\title{
Fractional Spectral Collocation Methods for Linear and Nonlinear Variable Order FPDEs
}

\author{
Mohsen Zayernouri and George Em Karniadakis* \\ Division of Applied Mathematics, Brown University, 182 George, Providence, RI 02912, USA
}

\begin{abstract}
While several high-order methods have been developed for fractional PDEs (FPDEs) with fixed order, there are no such methods for FPDEs with field-variable order. These equations allow multiphysics simulations seamlessly, e.g. from diffusion to sub-diffusion or from wave dynamics transitioning to diffusion, by simply varying the fractional order as a function of space or time. We develop an exponentially accurate fractional spectral collocation method for solving linear/nonlinear FPDEs with field-variable order. Following the spectral theory, developed in [1] for fractional Sturm-Liouville eigenproblems, we introduce a new family of interpolants, called left-/right-sided and central fractional Lagrange interpolants. We employ the fractional derivatives of (left-/right-sided) Riemann-Liouville and Riesz type and obtain the corresponding fractional differentiation matrices by collocating the field-variable fractional orders. We solve several FPDEs including timeand space-fractional advection-equation, time- and space- fractional advectiondiffusion equation, and finally the space-fractional Burgers' equation to demonstrate the performance of the method. In addition, we develop a spectral penalty method for enforcing inhomogeneous initial conditions. Our numerical results confirm the exponential-like convergence of the proposed fractional collocation methods.
\end{abstract}

Keywords: Jacobi polyfractonomial, space-time fractional Lagrange interpolants, variable-order differentiation matrix, Riemann-Liouville, Riesz and Caputo fractional derivatives, penalty method

${ }^{*}$ Corresponding author: george_karniadakis @ brown.edu, Fax: (401) 863-2722 


\section{Introduction}

The theory of fractional differential operators generalizes the notion of standard operators of integer orders to fractional orders. Such differential operators appear in modeling diverse physical problems involving e.g., porous or fractured media [2], viscoelastic materials [3], viscous fluid flows subject to wall-friction effects [4, 5, 6], bioengineering applications [7], and anomalous transport [8, 9, 10]. The notion of fractional derivatives has been rapidly extended to a variety of fractional partial differential equations (FPDEs) such as fractional Burgers' equation [11], Fokker-Planck equation [12], and advection-diffusion equation [13].

Recently, it has been demonstrated that in many dynamic processes, the underlying differential operators not only appear as fractional, but they also possess a dynamic nature in a sense that their order is field-variable, which may vary in time and/or space. For instance, several classes of random processes with variableorder fractional transition probability densities on unbounded domains have been studied in $[14,15]$. Moreover, the notion of variable-order fractional calculus has been used to dynamic modelling of heterogeneous physical systems. Examples are linear and nonlinear oscillators with viscoelastic damping by Coimbra [16], processing of geographical data using variable-order derivatives by Cooper and Cowan [17], signature verification through variable/adaptive fractional order differentiators by Tseng [18], constitutive laws in viscoelastic continuum mechanics by Ramirez et.al., [19], modeling of diffusive-convective effects on the oscillatory flows [20], anomalous diffusion problems by Sun et al. [21], fractional advectiondiffusion problem by Chen et al., [22, 23], mobile-immobile advection-dispersion model by Zhang et al. [24], and chloride ions sub-diffusion in concrete structures by Chen et. al. [25]. This approach opens up great opportunities for modeling and simulation of multiphysics phenomena, e.g. seamless transition from wave propagation to diffusion, or from local to non-local dynamics.

Such an extension from fixed-order to variable-order operators provides an invaluable prospect in modeling complex phenomena, whose behaviour otherwise may not be properly understood. For instance, in Fig. 1 (right), we investigate the decaying solution to the following time- and space fractional diffusion problem

$$
{ }_{0}^{C} \mathcal{D}_{t}^{\zeta(x)} u=\frac{\partial^{1+v} u}{\partial|x|^{1+v}},
$$

subject to $u(x, 0)=\left(1-x^{2}\right)$ and $u( \pm 1, t)=0$, in the absence of any external forces. In (1), ${ }_{0}^{C} \mathcal{D}_{t}^{\zeta}(\cdot)$ and $\frac{\partial^{1+v}(\cdot)}{\partial|x|^{1+v}}$ are fractional derivatives of Caputo and Riesz type, see 

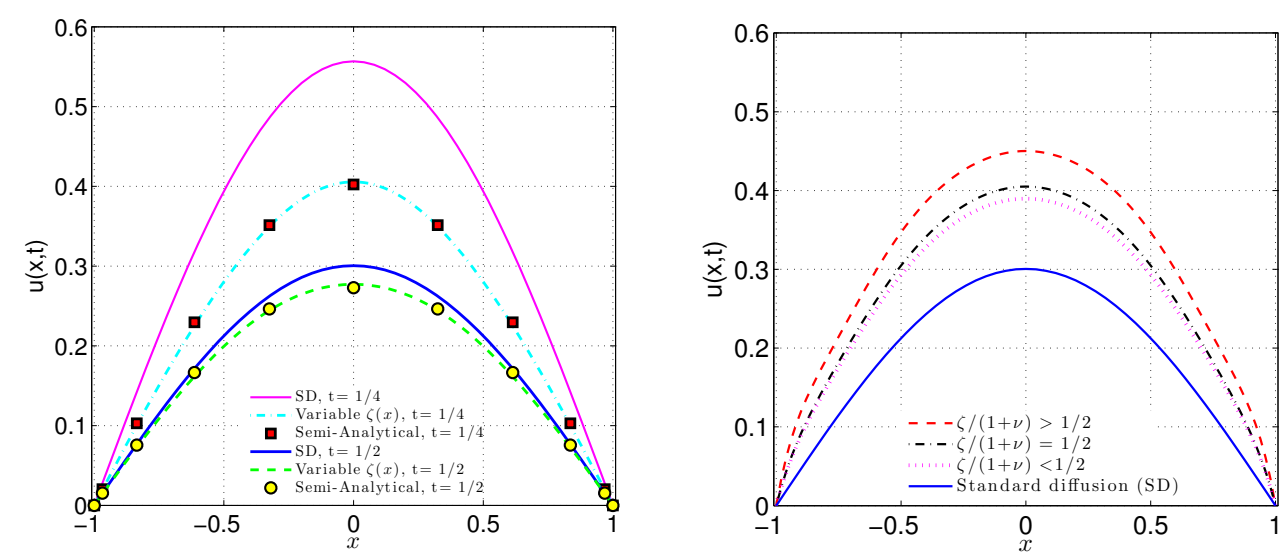

Figure 1: Variable-order (Left) versus fixed-order diffusion (Right). The initial condition is $u(x, 0)=1-x^{2}$ and the solutions are obtained at $t=\frac{1}{2}$, where the space-fractional order is $(1+v)=1.99$. While the fixed-order cases on the right plot exhibit the expected sub-diffusion process compared to the standard diffusion (SD) problem (i.e., when $\zeta=1$ and $1+v=2$ ), the variable-order test-case when $\zeta(x)=1 /(3|x|+11 / 10)$ on the left plot exhibit, surprisingly, a super-diffusion behaviour.

section 2. Here, we set $\zeta$ and $v \in(0,1)$, highlighting the anomalous sub-diffusion character of the problem, compared to the standard diffusion denoted by $S D$, i.e., when $\zeta=v=1$. We demonstrate the corresponding sub-diffusive behaviour in (1) by taking different but fixed values of $\zeta$ and $v$ and plotting the results in Fig. 1 (right) at $t=1 / 2$. As expected, the corresponding curves all lag behind the standard diffusion. Surprisingly, when we allow the temporal order $\zeta$ to vary across the domain, as shown in Fig. 1 (left) at $t=1 / 4$ and $t=1 / 2$, we observe a super-diffusive behaviour even for $\zeta(x) \in(0,1)$; here, $\zeta(x)=1 /(3|x|+11 / 10)$. In addition, we notice a more pronounced super-diffusion at earlier times in Fig. 1 (left). However, after a long time, the variable-order case becomes a sub-diffusion process.

The numerical approximation of variable-order FPDEs has been mostly developed using finite-difference methods (FDMs) (see [26, 27, 28, 29, 30] and references therein). Although easier to implement, the main challenge in FDM schemes is their limited accuracy interwoven with their inherent local character, while fractional derivatives are essentially global (nonlocal) differential operators. Hence, global schemes such as Spectral Methods (SM) may be more appropriate for discretizing fractional operators.

The idea of developing spectral methods for fixed order FODEs/FPDEs has 
received great attention over the last decade. Lin and $\mathrm{Xu}$ [31] developed a hybrid scheme for time-fractional diffusion problem; they also developed a time-space SM for time-fractional diffusion [32, 33]. Khader [34] proposed a Chebyshev collocation method for a space-fractional diffusion equation; while Piret and Hanert developed a radial basis function method for fractional diffusion equations [35]. Moreover, a Chebyshev spectral method [36], a Legendre spectral method [37], and an adaptive pseudospectral method [38] were proposed for solving fractional boundary value problems. In addition, generalized Laguerre spectral algorithms and Legendre spectral Galerkin method were developed by Baleanu et al. [39] and by Bhrawy and Alghamdi [40] for fractional initial value problems. Recently, Deng and Hesthaven [41] developed local discontinuous Galerkin methods for fractional diffusion equations, and $\mathrm{Xu}$ and Hesthaven [42] developed a stable multi-domain spectral penalty method for FPDEs. In all the aforementioned spectral methods, polynomial bases have been employed.

Recently, Zayernouri and Karniadakis [43, 44] and Zayernouri et.al. [45] developed spectrally accurate Petrov-Galerkin spectral and spectral element methods for non-delay and delay fractional differential equations in addition to the fractional advection equation, where they employed a new family of fractional bases, called Jacobi Polyfractonomials.

They introduced these polyfractonomials as the eigenfunctions of fractional Sturm-Liouville problems in [1], explicitly given as

$$
\text { (1) } \mathcal{P}_{n}^{\alpha, \beta, \mu}(\xi)=(1+\xi)^{-\beta+\mu-1} P_{n-1}^{\alpha-\mu+1,-\beta+\mu-1}(\xi), \quad \xi \in[-1,1]
$$

with $\mu \in(0,1),-1 \leq \alpha<2-\mu$, and $-1 \leq \beta<\mu-1$, representing the eigenfunctions of the singular FSLP of first kind (SFSLP-I), and

$$
{ }^{(2)} \mathcal{P}_{n}^{\alpha, \beta, \mu}(\xi)=(1-\xi)^{-\alpha+\mu-1} P_{n-1}^{-\alpha+\mu-1, \beta-\mu+1}(\xi), \quad \xi \in[-1,1],
$$

where $-1<\alpha<\mu-1$ and $-1<\beta<2-\mu$, and $\mu \in(0,1)$, denoting the eigenfunctions of the singular FSLP of second kind (SFSLP-II). Moreover, they employed these fractional bases to introduce a new class of fractional interpolants to develop efficient and spectrally accurate collocation methods in [46] for a variety of FODEs and FPDEs including multi-term FPDEs and the nonlinear space-fractional Burgers' equation. Employing the Jacobi polyfractonomials, Zayernouri and Karniadakis have recently developed a unified Petrov-Galerkin spectral method along with a unified fast solver in [47] that efficiently treats the whole family of elliptic, parabolic, and hyperbolic FPDEs in high-dimensions with spectral accuracy and in a unified fashion. 
To the best of our knowledge, no high-order or spectral methods have been developed for variable-order FPDEs up to date. The main contribution of the present study is the development of a new class of spectrally accurate fractional spectral collocation method for solving linear and nonlinear field-variable order in-time and in-space FPDEs. To this end, we introduce the left-/right-sided fractional Lagrange interpolants (FLIs) when spatial Riemann-Liouville derivatives are employed, in addition to the central FLIs when spatial Riesz derivatives are employed.

The organization of the paper is as follows: in section 2 we first present some preliminaries from fractional calculus. In section 3, we define the general setting of the model problem in this study, and the field-variable fractional order derivatives of Riemann-Liouville and Riesz type are introduced. In section 4, we construct three types of fractional Lagrange intrpolants and investigate their properties. Next in section 5, we obtain the associated differentiation matrices of variable-order, corresponding to each type of fractional derivatives. Subsequently, we investigate the performance of our fractional collocation spectral methods in the framework of linear and nonlinear variable-order FPDEs in section 6. Moreover in this section, we propose a penalty method to impose inhomogeneous initial conditions. We finally conclude the paper with a summary and discussion in section 7.

\section{Preliminaries}

We first provide some definitions from fractional calculus. Following [48], for a function $w(z) \in C^{n}\left[z_{L}, z_{R}\right]$, we denote by ${ }_{z_{L}} \mathcal{D}_{z}^{\gamma} w(z)$ the left-sided ReimannLiouville fractional derivative of order $\gamma$, when $n-1 \leq \gamma<n$, defined as

$$
{ }_{z_{L}}^{R L} \mathcal{D}_{z}^{\gamma} w(z)=\frac{1}{\Gamma(n-\gamma)} \frac{d^{n}}{d z^{n}} \int_{z_{L}}^{z} \frac{w(s)}{(z-s)^{\gamma+1-n}} d s, \quad z \in\left[z_{L}, z_{R}\right]
$$

where $\Gamma$ represents the Euler gamma function, and as $\gamma \rightarrow n$, the global operator ${ }_{z_{L}}^{R L} \mathcal{D}_{z}^{\gamma} \rightarrow d^{n} / d z^{n}$, recovering the local $n$-th order derivative with respect to $z$. We also denote by ${ }_{z}^{R L} \mathcal{D}_{z R}^{\gamma} w(z)$ the corresponding right-sided Reimann-Liouville fractional derivative of order $n-1 \leq \gamma<n$, defined as

$$
{ }_{z}^{R L} \mathcal{D}_{z_{R}}^{\gamma} w(z)=\frac{1}{\Gamma(n-\gamma)}(-1)^{n} \frac{d^{n}}{d z^{n}} \int_{z}^{b} \frac{w(s)}{(s-z)^{\gamma+1-n}} d s, \quad z \in\left[z_{L}, z_{R}\right] .
$$

Similarly, as $\gamma \rightarrow n$, the right-sided fractional derivative tends to the standard $n$-th order local derivative. We also recall from [48] a useful property of the Riemann- 
Liouville fractional derivatives. Assume that $0<p \leq 1,0<q \leq 1$ and $z>z_{L}$, then

$$
{ }_{z_{L}}^{R L} \mathcal{D}_{z}^{p+q} w(z)=\left({ }_{z_{L}}^{R L} \mathcal{D}_{z}^{p}\right)\left({ }_{z_{L}}^{R L} \mathcal{D}_{z}^{q}\right) w(z)=\left({ }_{z_{L}}^{R L} \mathcal{D}_{z}^{q}\right)\left({ }_{z_{L}}^{R L} \mathcal{D}_{z}^{p}\right) w(z)
$$

when $w\left(z_{L}\right)=0$, and

$$
{ }_{z}^{R L} \mathcal{D}_{z R}^{p+q} w(z)=\left({ }^{R L} \mathcal{D}_{z R}^{p}\right)\left({ }^{R L} \mathcal{D}_{z R}^{q}\right) w(z)=\left({ }^{R L} \mathcal{D}_{z R}^{q}\right)\left({ }_{z}^{R L} \mathcal{D}_{z R}^{p}\right) w(z)
$$

when $w\left(z_{R}\right)=0$. Moreover, for $k>-1$, we have

$$
{ }_{z_{L}}^{R L} \mathcal{D}_{z}^{\gamma}\left(z+z_{L}\right)^{k}=\frac{\Gamma(k+1)}{\Gamma(k+1-\gamma)}\left(z+z_{L}\right)^{k-\gamma}
$$

and

$$
{ }_{z}^{R L} \mathcal{D}_{z_{R}}^{\gamma}\left(z_{R}-z\right)^{k}=\frac{-\Gamma(k+1)}{\Gamma(k+1-\gamma)}\left(z_{R}-z\right)^{k-\gamma}
$$

if $\gamma \in(0,1)$. In addition, let again $z=\frac{z_{R}+z_{L}}{2}+\frac{z_{R}-z_{R}}{2} \xi$ map $x \in\left[x_{L}, x_{R}\right]$ with $\xi \in$ $[-1,1]$, known as the standard domain, to $\left[z_{L}, z_{R}\right]$. Then,

$$
{ }_{z_{L}}^{R L} \mathcal{D}_{z}^{\gamma} w(z)=\left(\frac{2}{z_{R}-z_{L}}\right)^{\gamma}{ }_{-1}^{R L} \mathcal{D}_{\xi}^{\gamma} w(z(\xi))
$$

moreover,

$$
{ }_{z}^{R L} \mathcal{D}_{z_{R}}^{\gamma} w(z)=\left(\frac{2}{z_{R}-z_{L}}\right)^{\gamma}{ }_{\xi}^{R L} \mathcal{D}_{1}^{\gamma} w(z(\xi))
$$

which are useful in the derivation of the emerging differentiation matrices in our method.

The corresponding fractional derivatives of Caputo type, i.e., ${ }_{z_{R}}^{C} \mathcal{D}_{z}^{\gamma} w(z)$ and ${ }_{z}^{C} \mathcal{D}_{z_{R}}^{\gamma} w(z)$, are also defined by interchanging the order of differentiation and integration in (4) and (5) as

$$
{ }_{z_{L}}^{C} \mathcal{D}_{z}^{\gamma} w(z)=\frac{1}{\Gamma(n-\gamma)} \int_{z_{L}}^{z} \frac{\frac{d^{n} w(s)}{d s^{n}}}{(z-s)^{\gamma+1-n}} d s, \quad z \in\left[z_{L}, z_{R}\right]
$$

and

$$
{ }_{z}^{C} \mathcal{D}_{z_{R}}^{\gamma} w(z)=\frac{(-1)^{n}}{\Gamma(n-\gamma)} \int_{z}^{b} \frac{\frac{d^{n} w(s)}{d s^{n}}}{(s-z)^{\gamma+1-n}} d s, \quad z \in\left[z_{L}, z_{R}\right]
$$


which we will employ in FPDEs subject to non-homogeneous initial conditions. From the definition of the Caputo derivatives, the properties (8) and (9) become

$$
{ }_{z_{L}}^{C} \mathcal{D}_{z}^{\gamma}\left(z+z_{L}\right)^{k}= \begin{cases}0, & k<\gamma, \\ \frac{\Gamma(k+1)}{\Gamma(k+1-\gamma)}\left(z+z_{L}\right)^{k-\gamma}, & 0<\gamma \leq k\end{cases}
$$

and

$$
{ }_{z}^{C} \mathcal{D}_{z_{R}}^{\gamma}\left(z_{R}-z\right)^{k}= \begin{cases}0, & k<\gamma, \\ \frac{-\Gamma(k+1)}{\Gamma(k+1-\gamma)}\left(z_{R}-z\right)^{k-\gamma}, & 0<\gamma \leq k .\end{cases}
$$

We denote by $\frac{\partial^{\gamma} u}{\partial|z|^{\gamma}}$ the Riesz fractional derivative of order $\gamma$, when $n-1<\gamma<n$, defined in terms of left- and right-sided Riemann-Liouville fractional derivatives as

$$
\frac{d^{\gamma} u}{d|z|^{\gamma}}=C_{\gamma}\left({ }_{z_{L}}^{R L} \mathcal{D}_{z}^{\gamma} w(z)+{ }_{z}^{R L} \mathcal{D}_{z R}^{\gamma} w(z)\right)
$$

where $C_{\gamma}=-\frac{1}{2} \sec (\gamma \pi / 2)$. It is easy to check that as $\gamma \rightarrow n$, asymptotically $\frac{d^{\gamma}(\cdot)}{d|z|^{\gamma}} \rightarrow$ $d^{n}(\cdot) / d z^{n}$.

So far, we note that all the aforementioned fractional derivatives are of a constant order $\gamma$ and are defined originally for the univariate function $w(z)$. In the sequel, we introduce a model problem, in which the fractional operators are defined through generalization of the given definitions to multi-variate functions, in which the corresponding fractional orders are field variables rather than constants.

\section{Problem Definition}

Let $\zeta, \sigma$ and $v: \mathbb{R}^{2} \rightarrow \mathbb{R}$ be continuous functions. We study the following nonlinear variable-order time- and space-fractional FPDE for all $(x, t) \in[a, b] \times$ $[0, T]$ as

$$
\begin{aligned}
{ }_{0}^{R L} \mathcal{D}_{t}^{\zeta(x, t)} u+g(u)^{*} \mathcal{D}_{x}^{\sigma(x, t)} u & =K^{*} \mathcal{D}_{x}^{1+v(x, t)} u+f(u ; x, t) \\
u(a, t) & =u(b, t)=0 \\
u(x, 0) & =0
\end{aligned}
$$

where $K>0$ and $f(u ; x, t)$ denotes the forcing- and/or reaction term. Moreover, the temporal order $\zeta(x, t) \in(0,1)$, the spatial advection order $\sigma(x, t), \in(0,1)$, and 
the spatial diffusion order $1+\mathrm{v}(x, t) \in(1,2)$, i.e., $\mathrm{v}(x, t) \in(0,1)$. We shall discuss the regularity of the aforementioned field variable orders in section 6. Here, ${ }_{0}^{R L} \mathcal{D}_{t}^{\zeta(x, t)} u$ represents the $\zeta(x, t)$-th order left-sided partial time derivative of $u(x, t)$ of Riemann-Liouville type, defined as

$$
{ }_{0}^{R L} \mathcal{D}_{t}^{\zeta(x, t)} u(x, t)=\frac{1}{\Gamma[1-\zeta(x, t)]} \frac{\partial}{\partial t} \int_{0}^{t} \frac{u(x, s) d s}{(t-s)^{\zeta(x, t)}},
$$

based on (4). We consider the space-fractional derivatives ${ }^{*} \mathcal{D}_{x}$, associated with the advection and the diffusion terms in (17), to be of either (i) Riemann-Liouville or (ii) Riesz type, when homogeneous initial/boundary conditions are imposed. Alternatively, when inhomogeneous initial conditions are enforced, we consider the temporal derivative to be of Caputo type.

(I) ${ }^{*} \mathcal{D}_{x}$ of Riemann-Liouville type We define the corresponding advection partial fractional derivative ${ }^{*} \mathcal{D}_{x}^{\sigma(x, t)} u$ of Riemann-Liouville type according to (4) and (5) and based on the direction of the transport velocity as

$$
{ }^{*} \mathcal{D}_{x}^{\sigma(x, t)} u(x, t) \equiv \begin{cases}{ }_{a}^{R L} \mathcal{D}_{x}^{\sigma(x, t)} u=\frac{1}{\Gamma[1-\sigma(x, t)]} \frac{\partial}{\partial x} \int_{a}^{x} \frac{u(r, t) d r}{(x-r)^{\sigma(x, t)},} & g>0, \\ { }_{x}^{R L} \mathcal{D}_{b}^{\sigma(x, t)} u=\frac{1}{\Gamma[1-\sigma(x, t)]}\left(-\frac{\partial}{\partial x}\right) \int_{x}^{b} \frac{u(r, t) d r}{(r-x)^{\sigma(x, t)}}, & g<0 .\end{cases}
$$

In addition, we define the diffusion partial fractional derivative ${ }^{*} \mathcal{D}_{x}^{1+v(x, t)} u$ of Riemann-Liouville type either as ${ }_{a}^{R L} \mathcal{D}_{x}^{1+v(x, t)} u \equiv \frac{\partial}{\partial x}\left[{ }_{a}^{R L} \mathcal{D}_{x}^{v(x, t)} u\right]$ or ${ }_{x}^{R L} \mathcal{D}_{b}^{1+v(x, t)} u \equiv$ $\frac{\partial}{\partial x}\left[{ }_{x}^{R L} \mathcal{D}_{b}^{v(x, t)} u\right]$.

(II) ${ }^{*} \mathcal{D}_{x}$ of Riesz type We alternatively define the corresponding advection partial fractional derivative ${ }^{*} \mathcal{D}_{x}^{\sigma(x, t)} u$ of Riesz type as

$$
{ }^{*} \mathcal{D}_{x}^{\sigma(x, t)} u(x, t) \equiv \frac{\partial^{\sigma(x, t)} u}{\partial|x|^{\sigma(x, t)}}=C_{\sigma(x, t)}\left({ }_{a}^{R L} \mathcal{D}_{x}^{\sigma(x, t)} u+{ }_{x}^{R L} \mathcal{D}_{b}^{\sigma(x, t)} u\right) .
$$

Moreover, from (6) and (7), we define the diffusion partial fractional derivative ${ }^{*} \mathcal{D}_{x}^{1+v(x, t)} u$ to be of Riesz type as

$$
{ }^{*} \mathcal{D}_{x}^{1+v(x, t)} u(x, t)=C_{1+v(x, t)} \frac{\partial}{\partial x}\left[{ }_{a}^{R L} \mathcal{D}_{x}^{v(x, t)} u(x, t)+{ }_{x}^{R L} \mathcal{D}_{b}^{v(x, t)} u(x, t)\right] .
$$

In the following, we develop a fractional spectral collocation method for efficient solution of (17) based on the variable-order fractional differential operators defined here. 


\section{Fractional Lagrange Interpolants (FLIs)}

We define a set of interpolation points on which the corresponding Lagrange interpolants are obtained. Specifically, we employ a new family of left- and rightsided space-time fractional Lagrange interpolants (FLI) rather than utilizing the standard algebraic/trigonometric polynomial Lagrange basis functions. We shall demonstrate that such a construction leads to efficient computation of the corresponding differentiation matrices in addition to efficient approximations. Denoting by $u_{N}$ an approximation of the solution in terms of such interpolators, we formulate our collocation method by requiring the residual of the problem i.e.,

$$
R_{N}(x, t)={ }_{0}^{R L} \mathcal{D}_{t}^{\zeta(x, t)} u_{N}+g(u)^{*} \mathcal{D}_{x}^{\sigma(x, t)} u_{N}-K^{*} \mathcal{D}_{x}^{1+v(x, t)} u_{N}-f\left(u_{N} ; x, t\right),
$$

to vanish on the same set of grid points called collocation points.

Our fractional spectral collocation scheme is inspired by a new spectral theory developed for fractional Sturm-Liouville eigen-problems (FSLP) in [1]. The idea is to represent the solution to (17) in terms of new fractional (non-polynomial) basis functions, called Jacobi polyfractonomials, which are the eigenfunctions of the FSLP of first and second kind, explicitly given in (2) and (3). So, depending on the choice of the spatial fractional derivative ${ }^{*} \mathcal{D}_{x}$ in (17), we introduce the corresponding fractional Lagrange interpolants.

\subsection{Construction of FLI when ${ }^{*} \mathcal{D}_{x} \equiv{ }^{R L} \mathcal{D}_{x}$}

Let $\alpha=\beta=-1$ in (2) and (3), which corresponds to the regular eigenfunctions of first and second kind, given as

$$
{ }^{(1)} \mathcal{P}_{n}^{\mu}(\xi)=(1+\xi)^{\mu} P_{n-1}^{-\mu, \mu}(\xi), \quad \xi \in[-1,1],
$$

and

$$
{ }^{(2)} \mathcal{P}_{n}^{\mu}(\xi)=(1-\xi)^{\mu} P_{n-1}^{\mu,-\mu}(\xi), \quad \xi \in[-1,1] .
$$

where we recall that $\mu \in(0,1)$. From the properties of the eigensolutions in [1], the left-sided fractional derivatives of (25) and (26) are given as

$$
{ }_{-1}^{R L} \mathcal{D}_{\xi}^{\mu}\left({ }^{(1)} \mathcal{P}_{n}^{\mu}(\xi)\right)={ }_{\xi}^{R L} \mathcal{D}_{1}^{\mu}\left({ }^{(2)} \mathcal{P}_{n}^{\mu}(\xi)\right)=\frac{\Gamma(n+\mu)}{\Gamma(n)} P_{n-1}(x),
$$

where $P_{n-1}(x)$ denotes a Legendre polynomial of order $(n-1)$. Hence, in general, we can employ the univariate eigenfunctions (25) and (26) to construct the spacetime modal basis functions needed. However, here we alternatively construct suitable nodal basis functions based on the transport velocity and the corresponding choice of the spatial derivatives ${ }_{a}^{R L} \mathcal{D}_{x}$ or ${ }_{x}^{R L} \mathcal{D}_{b}$; see (21). 


\subsubsection{Left-Sided FLIs when ${ }^{*} \mathcal{D}_{x} \equiv{ }_{a}^{R L} \mathcal{D}_{x}$}

We seek solutions as a nodal expansion

$$
u_{N}(x, t)=\sum_{m=1}^{\mathcal{M}} \sum_{n=1}^{\mathcal{N}} u_{N}\left(x_{m}, t_{n}\right) L_{m}^{\mu}(x) \mathcal{T}_{n}^{\tau}(t)
$$

where $L_{m}^{\mu}(x)$ represent the left-sided spatial FLIs and $\mathcal{T}_{n}^{\tau}(t)$ denote the temporal FLIs, which are defined on some interpolations points $a=x_{1}<x_{2}<\cdots<x_{\mathscr{M}}=b$ and $0=t_{1}<t_{2}<\cdots<t_{\mathcal{N}}=T$ as

$$
L_{m}^{\mu}(x)=\left(\frac{x-x_{1}}{x_{m}-x_{1}}\right)^{\mu} \prod_{\substack{k=1 \\ k \neq m}}^{\mathcal{M}}\left(\frac{x-x_{k}}{x_{m}-x_{k}}\right), 2 \leq m \leq \mathcal{M}-1
$$

all of fractional order $(\mathcal{M}+\mu-1)$, and

$$
\mathcal{T}_{n}^{\tau}(t)=\left(\frac{t}{t_{n}}\right)^{\tau} \prod_{\substack{q=1 \\ q \neq n}}^{\mathcal{N}}\left(\frac{t-t_{q}}{t_{n}-x_{q}}\right), 2 \leq n \leq \mathcal{N},
$$

of fractional order $(\mathcal{N}+\tau-1)$. Here, we call the superscript $\mu$ and $\tau$ as spatial and temporal interpolation parameters, respectively. We set these constant fractional parameters prior to solving (17) from the variable orders given, i.e., $\tau, \sigma$, and $v$. Moreover, we note that the fractional interpolants satisfy the Kronecker delta property, i.e., $H_{m}^{\mu}\left(x_{k}\right)=\delta_{k m}$ and $L_{n}^{\tau}\left(t_{q}\right)=\delta_{q n}$, at interpolation points.

Because of the homogeneous Dirichlet boundary/initial condition(s) in (17), we only construct $L_{m}^{\mu}(x)$ for $m=2,3, \cdots, \mathcal{M}$ when the maximum fractional order $1+v \in(1,2)$, where we set $u_{N}\left(x_{1}, t\right)=u_{N}\left(x_{\mathfrak{M}}, t\right)=0$. Moreover, when $\tau \in(0,1)$, there are only $(\mathcal{N}-1)$ fractional Lagrange interpolants $\mathcal{T}_{n}^{\tau}(t), n=2,3, \cdots, \mathcal{N}$, since we impose $u_{N}\left(x, t_{1}\right)=0$.

\subsubsection{Right-Sided FLIs when ${ }^{*} \mathcal{D}_{x} \equiv{ }_{x}^{R L} \mathcal{D}_{b}$}

In this case, we seek the solution as another nodal expansion given by

$$
u_{N}(x, t)=\sum_{m=1}^{\mathcal{M}} \sum_{n=1}^{\mathcal{N}} u_{N}\left(x_{m}, t_{n}\right) R_{m}^{\mu}(x) \mathcal{T}_{n}^{\tau}(t)
$$


where $R_{m}^{\mu}(x)$ represent the right-sided spatial FLIs, defined on the interpolations points as

$$
R_{m}^{\mu}(x)=\left(\frac{x_{\mathcal{M}}-x}{x_{\mathcal{M}}-x_{m}}\right)^{\mu} \prod_{\substack{k=1 \\ k \neq m}}^{\mathcal{M}}\left(\frac{x-x_{k}}{x_{m}-x_{k}}\right), 2 \leq m \leq \mathcal{M}-1,
$$

which are all again of fractional order $(\mathcal{M}+\mu-1)$.

\subsection{Central FLIs when ${ }^{*} \mathcal{D}_{x} \equiv \frac{\partial}{\partial|x|}$ of Riesz Type}

When the fractional derivatives in (17) are all of Riesz type, we seek the solution as

$$
u_{N}(x, t)=\sum_{m=1}^{\mathcal{M}+1} \sum_{n=1}^{\mathcal{N}} u_{N}\left(x_{m}, t_{n}\right) h_{m}(x) \mathcal{T}_{n}^{\tau}(t),
$$

where $h_{m}(x)$ represent the standard polynomial Lagrange interpolants, defined on the interpolations points as

$$
h_{m}(x)=\prod_{\substack{k=1 \\ k \neq m}}^{\mathcal{M}+1}\left(\frac{x-x_{k}}{x_{m}-x_{k}}\right), 2 \leq m \leq \mathcal{M},
$$

which are all of order $\mathcal{M}$. The polynomial choice of $h_{m}(x)$ is mainly due to the $c o$ existence of the left- and right-sided fractional derivatives in the definition of the Riesz derivatives in (22) and (23). Hence, compared to the structure of the FLIs in (29) and (32), we call the FLIs presented in (33) central in-space interpolants. In fact, they can be viewed as the nodal representations of Legendre polynomials i.e., $P_{\mathscr{M}}(x)$, which simultaneously appear to be the regular eigenfucntions (25) and (26) asymptotically when $\mu \rightarrow 0$ setting $n=\mathcal{M}+1$.

When the time-derivative is of Caputo type, which is a proper setting in which we can enforce the inhomogeneous initial conditions, we also use the standard Legendre bases in time, i.e.,

$$
u_{N}(x, t)=\sum_{m=1}^{\mathcal{M}+1} \sum_{n=1}^{\mathcal{N}+1} u_{N}\left(x_{m}, t_{n}\right) h_{m}(x) h_{n}(t)
$$

where

$$
h_{n}(t)=\prod_{\substack{k=1 \\ k \neq n}}^{\mathcal{N}+1}\left(\frac{t-t_{k}}{t_{m}-t_{k}}\right), 1 \leq n \leq \mathcal{N}+1,
$$

which allows us to properly penalize the initial condition to the problem and develop a stable scheme. 


\section{Fractional Differentiation Matrices}

We derive the corresponding spatial and temporal fractional differentiation matrices assuming that the collocation and interpolation points coincide. We first choose the suitable expansion among those given in (28), (31), or (33) based on the choice of the spatial fractional derivative ${ }^{*} \mathcal{D}_{x}$. Then, we derive the corresponding differentiation matrices.

\section{1. ${ }^{*} \mathcal{D}_{x}$ of Left-Sided Riemann-Liouville Type}

Whether left- or right-sided Riemann-Liouville derivatives are employed in (17), we obtain the corresponding to left- or right-sided fractional differentiation matrices of order $\sigma(x, t)$ and $1+\mathrm{v}(x, t)$.

Theorem 5.1. Let $\sigma=\sigma(x, t) \in C([a, b] \times[0, T])$ and consider the affine mapping that maps $\xi \in[-1,1]$ to $x \in[a, b]$. Then, when ${ }^{*} \mathcal{D}_{x} \equiv{ }_{a}^{R L} \mathcal{D}_{x}$ in (17), the left-sided spatial differentiation matrix ${ }^{R L} D_{L}^{\sigma}$ of Riemann-Liouville type, corresponding to the nodal FLI expansion (28), is a three-dimensional matrix whose entries are given by

$$
\left\{{ }^{R L} \boldsymbol{D}_{L}^{\sigma}\right\}_{i k m}=\left(\frac{2}{b-a}\right)^{\sigma\left(x_{i}, t_{k}\right)} A_{m} \sum_{j=1}^{\mathcal{M}} \beta_{m j}^{L} F_{j}^{L, \sigma}\left(x_{i}, t_{k}\right)
$$

where $i, m=2,3, \cdots, \mathcal{M}, k=2,3, \cdots, \mathcal{N}$, also $F_{j}^{L, \sigma}(x(\xi), t)$ is explicitly given as

$$
F_{j}^{L, \sigma}(x(\xi), t)=\sum_{q=0}^{j-1} b_{j q}^{\mu}(1+\xi)^{q+\mu-\sigma},
$$

in which $A_{m}=\left(\frac{b-a}{2 x_{m}-2 a}\right)^{\mu}$, and finally $\beta_{m j}^{L}$ and $b_{j q}^{\mu}$ are the corresponding expansion coefficients, given a priori by (A.1) and (A.7).

Proof. Given in Appendix A.

Remark 5.2. In standard collocation methods applied to constant/integer-order operators, the corresponding differentiation matrices are two-dimensional. The extra dimension appearing in the left-sided differentiation matrix ${ }^{R L} \mathbf{D}_{L}^{\sigma}$ is due to the field-variable $\sigma(x, t)$, which makes the corresponding fractional differential operator vary across the computational domain. Consequently, by collocating the 
fractional order on each collocation point $\left(x_{i}, t_{k}\right)$, we must compute all the entries associated with the whole set of the spatial points indexed by " $m$ ". Therefore, it naturally renders the corresponding differentiation matrix three-dimensional. Interestingly, when $\sigma=\sigma(x)$, we reduce the dimension of ${ }^{R L} \mathbf{D}_{L}^{\sigma}$ by one, and we obtain the entries of the two-dimensional differentiation matrix as

$$
\left\{{ }^{R L} \mathbf{D}_{L}^{\sigma}\right\}_{i m}=\left(\frac{2}{b-a}\right)^{\sigma\left(x_{i}\right)} A_{m} \sum_{j=1}^{\mathcal{M}} \beta_{m j}^{L} F_{j}^{L, \sigma}\left(x_{i}\right) .
$$

Moreover, when $\sigma$ is constant, the differentiation matrix in (39) is further reduced to

$$
\left\{{ }^{R L} \mathbf{D}_{L}^{\sigma}\right\}_{i m}=\left(\frac{2}{b-a}\right)^{\sigma} A_{m} \sum_{j=1}^{\mathcal{M}} \beta_{m j}^{L} \frac{\Gamma(j+\sigma)}{\Gamma(j)} P_{j-1}\left(\xi_{i}\right),
$$

previously given in [46], where $x_{i}=\frac{a+b}{2}+\frac{b-a}{2} \xi_{i}$.

The next theorem provides the corresponding left-sided differentiation matrix for the diffusion term in (17) when ${ }^{*} \mathcal{D}_{x}^{1+v} \equiv{ }_{a}^{R L} \mathcal{D}_{x}^{1+v}$.

Theorem 5.3. Let $\mathrm{v}=\mathrm{v}(x, t) \in C([a, b] \times[0, T])$ and consider the affine mapping the maps $\xi \in[-1,1]$ to $x \in[a, b]$. Then, the left-sided spatial differentiation matrix ${ }^{R L} \boldsymbol{D}_{L}^{1+v}$ of Riemann-Liouville type, corresponding to the nodal FLI expansion (28) when ${ }^{*} \mathcal{D}_{x} \equiv{ }_{a}^{R L} \mathcal{D}_{x}$ in (17), is a three-dimensional matrix whose entries are given by

$$
\left\{{ }^{R L} \boldsymbol{D}_{L}^{1+v}\right\}_{i k m}=\left(\frac{2}{b-a}\right)^{1+v\left(x_{i}, t_{k}\right)} A_{m} \sum_{j=1}^{\mathcal{M}} \beta_{m j}^{L} \mathcal{F}_{j}^{L, v}\left(x_{i}, t_{k}\right),
$$

in which $A_{m}=\left(\frac{b-a}{2 x_{m}-2 a}\right)^{\mu}, i, m=2,3, \cdots, \mathcal{M}, k=2,3, \cdots, \mathcal{N}$, and $\mathcal{F}_{j}^{L, v}(x(\xi), t)$ is explicitly given by

$$
\begin{aligned}
\mathcal{F}_{j}^{L, v}(x(\xi), t) & =\mathbb{I}_{\{j \geq 1\}} \sum_{q=0}^{j-1} \mathbb{B}_{j q}^{\mu} \cdot(1+\xi)^{q+\mu-1-v\left(x_{i}, t_{k}\right)} \\
& +\mathbb{I}_{\{j \geq 2\}} \sum_{q=0}^{j-2} B_{j q}^{\mu} \cdot(1+\xi)^{q+\mu-v\left(x_{i}, t_{k}\right)}
\end{aligned}
$$

where $\mathbb{B}_{j q}^{\mu}$ and $B_{j q}^{\mu}$ are the corresponding expansion coefficients, given a priori by (B.4) and (B.5). 
Proof. Given in Appendix B.

In analogy with Remark 5.2, we also appreciate the appearance of the extra dimension in the right-sided differentiation matrix ${ }^{R L} \mathbf{D}_{L}^{1+v}$. Similarly, when $v=$ $v(x)$, we reduce the dimension of ${ }^{R L} \mathbf{D}_{L}^{1+v}$ by one and obtain the entries of the two-dimensional differentiation matrix as

$$
\left\{{ }^{R L} \mathbf{D}_{L}^{1+v}\right\}_{i m}=\left(\frac{2}{b-a}\right)^{1+v\left(x_{i}\right)} A_{m} \sum_{j=1}^{\mathcal{M}} \beta_{m j}^{L} \mathcal{F}_{j}^{L, v}\left(x_{i}\right)
$$

Remark 5.4. We note that the coefficients $\beta_{m j}^{L}$, shown in (A.1), are obtained only once and are utilized as many times as needed to construct ${ }^{R L} \mathbf{D}_{L}^{\sigma}$ and ${ }^{R L} \mathbf{D}_{L}^{1+v}$ for any order $\sigma, v \in(0,1)$.

\section{2. ${ }^{*} \mathcal{D}_{x}$ of Right-Sided Riemann-Liouville Type}

Following similar steps, presented in section 5.1, we now construct the corresponding right-sided advection differentiation matrix of order $\sigma(x, t)$ and the right-sided diffusion differentiation matrix of order $1+\mathrm{v}(x, t)$ when ${ }^{*} \mathcal{D}_{x} \equiv{ }_{x}{ }^{R} \mathcal{D}_{b}$ in the sequel.

Theorem 5.5. Let $\sigma=\sigma(x, t) \in C([a, b] \times[0, T])$ and consider the affine mapping that maps $\xi \in[-1,1]$ to $x \in[a, b]$. Then, when ${ }^{*} \mathcal{D}_{x} \equiv{ }_{x}^{R L} \mathcal{D}_{b}$ in (17), the right-sided spatial differentiation matrix ${ }^{R L} D_{R}^{\sigma}$ of Riemann-Liouville type, corresponding to the nodal FLI expansion (31), is a three-dimensional matrix whose entries are given by

$$
\left\{{ }^{R L} \boldsymbol{D}_{R}^{\sigma}\right\}_{i k m}=\left(\frac{2}{b-a}\right)^{\sigma\left(x_{i}, t_{k}\right)} \mathcal{A}_{m} \sum_{j=1}^{\mathcal{M}} \beta_{m j}^{R} F_{j}^{R, \sigma}\left(x_{i}, t_{k}\right)
$$

in which $i, m=2,3, \cdots, \mathcal{M}, k=2,3, \cdots, \mathcal{N}$, also $F_{j}^{R, \sigma}(x(\xi), t)$ is explicitly given as

$$
F_{j}^{R, \sigma}(x(\xi), t)=\sum_{q=0}^{j-1} c_{j q}^{\mu}(1-\xi)^{q+\mu-\sigma},
$$

in which $\mathcal{A}_{m}=1 /\left(\xi_{\mathcal{M}}-\xi_{m}\right)^{\mu}$, and finally $\beta_{m j}^{R}$ and $c_{j q}^{\mu}$ are the corresponding expansion coefficients, given a priori by (C.1) and (C.6). 
Proof. Given in Appendix C.

The next theorem provides the corresponding right-sided differentiation matrix for the diffusion term in (17) when ${ }^{*} \mathcal{D}_{x}^{1+v} \equiv{ }_{x}{ }_{x} \mathcal{D}_{b}^{1+v(x, t)}$.

Theorem 5.6. Let $\mathrm{v}=\mathrm{v}(x, t) \in C([a, b] \times[0, T])$ and consider the affine mapping that maps $\xi \in[-1,1]$ to $x \in[a, b]$. Then, the right-sided spatial differentiation matrix ${ }^{R L} \boldsymbol{D}_{R}^{1+v}$ of Riemann-Liouville type, corresponding to the nodal FLI expansion (31) when ${ }^{*} \mathcal{D}_{x} \equiv{ }_{x}^{R L} \mathcal{D}_{b}$ in (17), is a three-dimensional matrix whose entries are given by

$$
\left\{^{R L} \boldsymbol{D}_{R}^{1+v}\right\}_{i k m}=\left(\frac{2}{b-a}\right)^{1+v\left(x_{i}, t_{k}\right)} \mathcal{A}_{m} \sum_{j=1}^{\mathcal{M}} \beta_{m j}^{R} \mathcal{F}_{j}^{R, v}\left(x_{i}, t_{k}\right),
$$

where $i, m=2,3, \cdots, \mathcal{M}, k=2,3, \cdots, \mathcal{N}$, and $\mathcal{F}_{j}^{R, v}(x(\xi), t)$ is explicitly given by

$$
\begin{aligned}
\mathcal{F}_{j}^{R, v}(x(\xi), t) & =\mathbb{I}_{\{j \geq 1\}} \sum_{q=0}^{j-1} \mathbb{C}_{j q}^{\mu} \cdot(1-\xi)^{q+\mu-1-v\left(x_{i}, t_{k}\right)} \\
& +\mathbb{I}_{\{j \geq 2\}} \sum_{q=0}^{j-2} C_{j q}^{\mu} \cdot(1-\xi)^{q+\mu-v\left(x_{i}, t_{k}\right)}
\end{aligned}
$$

in which $C_{j q}^{\mu}$ and $\mathbb{C}_{j q}^{\mu}$ are the corresponding expansion coefficients, given a priori by (D.3) and (D.2).

Proof. Given in Appendix D.

\section{3. ${ }^{*} \mathcal{D}_{x}$ of Riesz Type}

The following Lemma is useful in the derivation and construction of the Riesz spatial differentiation matrices.

Lemma 5.7. [49] For $\mu>0, \alpha>-1, \beta>-1$, and $\forall \xi \in[-1,1]$

$$
(1+\xi)^{\beta+\mu} \frac{P_{n}^{\alpha-\mu, \beta+\mu}(\xi)}{P_{n}^{\alpha-\mu, \beta+\mu}(-1)}=\frac{\Gamma(\beta+\mu+1)}{\Gamma(\beta+1) \Gamma(\mu) P_{n}^{\alpha, \beta}(-1)} \int_{-1}^{\xi} \frac{(1+s)^{\beta} P_{n}^{\alpha, \beta}(s)}{(\xi-s)^{1-\mu}} d s,
$$


and

$$
(1-\xi)^{\alpha+\mu} \frac{P_{n}^{\alpha+\mu, \beta-\mu}(\xi)}{P_{n}^{\alpha+\mu, \beta-\mu}(+1)}=\frac{\Gamma(\alpha+\mu+1)}{\Gamma(\alpha+1) \Gamma(\mu) P_{n}^{\alpha, \beta}(+1)} \int_{\xi}^{1} \frac{(1-s)^{\alpha} P_{n}^{\alpha, \beta}(s)}{(s-\xi)^{1-\mu}} d s .
$$

By the definition of the left-sided Riemann-Liouville integral ${ }_{-1}^{R L} I_{\xi}^{\mu}$ and evaluating the special end-values $P_{n}^{\alpha-\mu, \beta+\mu}(-1)$ and $P_{n}^{\alpha, \beta}(-1)$, we can re-write (48) as

$$
{ }_{-1}^{R L} I_{\xi}^{\mu}\left\{(1+\xi)^{\beta} P_{n}^{\alpha, \beta}(\xi)\right\}=\frac{\Gamma(n+\beta+1)}{\Gamma(n+\beta+\mu+1)}(1+\xi)^{\beta+\mu} P_{n}^{\alpha-\mu, \beta+\mu}(\xi) .
$$

Now, by taking the fractional derivative ${ }_{-1}^{R L} \mathcal{D}_{\xi}^{\mu}$ on the when $\beta=-\mu$ and $\alpha=\mu$ we obtain

$$
{ }_{-1}^{R L} \mathcal{D}_{\xi}^{\mu}\left\{P_{n}(\xi)\right\}=\frac{\Gamma(n+1)}{\Gamma(n-\mu+1)}(1+\xi)^{-\mu} P_{n}^{\mu,,-\mu}(\xi) .
$$

Similarly, by the definition of the right-sided Riemann-Liouville integral ${ }_{\xi}^{R L} I_{1}^{\mu}$ and evaluating the special end-values $P_{n}^{\alpha-\mu, \beta+\mu}(+1)$ and $P_{n}^{\alpha, \beta}(+1)$, we can re-write (49) as

$$
{ }_{\xi}^{R L} I_{1}^{\mu}\left\{(1-\xi)^{\alpha} P_{n}^{\alpha, \beta}(\xi)\right\}=\frac{\Gamma(n+\alpha+1)}{\Gamma(n+\alpha+\mu+1)}(1-\xi)^{\alpha+\mu} P_{n}^{\alpha+\mu, \beta-\mu}(\xi) .
$$

In a similar fashion, by taking the fractional derivative ${ }_{\xi}^{R L} \mathcal{D}_{-1}^{\mu}$ on the both sides when $\alpha=-\mu$ and $\beta=\mu$ we obtain

$$
{ }_{\xi}^{L} \mathcal{D}_{1}^{\mu}\left\{P_{n}(\xi)\right\}=\frac{\Gamma(n+1)}{\Gamma(n-\mu+1)}(1-\xi)^{-\mu} P_{n}^{-\mu, \mu}(\xi) .
$$

Theorem 5.8. Let $\sigma=\sigma(x, t) \in C([a, b] \times[0, T])$ and consider the affine mapping that maps $\xi \in[-1,1]$ to $x \in[a, b]$. Then, when ${ }^{*} \mathcal{D}_{x} \equiv \partial^{\sigma(x, t)} u / \partial|x|^{\sigma(x, t)}$ in (17), the right-sided spatial differentiation matrix $\boldsymbol{D}_{\text {Riesz }}^{\sigma}$ of Riesz type, corresponding to the nodal FLI expansion (33), is a three-dimensional matrix whose entries are given by

$$
\left\{\boldsymbol{D}_{\text {Riesz }}^{\sigma}\right\}_{i k m}=\left[\left(\frac{2}{b-a}\right)^{\sigma(x, t)} C_{\sigma(x, t)}\right]{ }_{\left(x_{i}, t_{k}\right)} \sum_{j=1}^{\mathcal{M}} \widetilde{\beta}_{m j} z_{j}^{\sigma}\left(x_{k}, t_{k}\right),
$$


in which $i, m=2,3, \cdots, \mathcal{M}, k=2,3, \cdots, \mathcal{N}, \xi=2 \frac{x-a}{b-a}$, and $\widetilde{\beta}_{m j}$ are the corresponding expansion coefficients, given a priori by (E.1). Moreover, $Z_{j}^{\sigma}(x, t)$ is explicitly given as

$$
z_{j}^{\sigma}(x, t)=\frac{\Gamma(j+1)}{\Gamma\left(j-\sigma\left(x_{i}, t_{k}\right)+1\right)}\left[(1+\xi)^{-\sigma(x, t)} P_{j}^{\sigma,-\sigma}(\xi)+(1-\xi)^{-\sigma(x, t)} P_{j}^{-\sigma, \sigma}(\xi)\right],
$$

Proof. Given in Appendix E.

Theorem 5.9. Let $\mathrm{v}=\mathrm{v}(x, t) \in C([a, b] \times[0, T])$ and consider the affine mapping that maps $\xi \in[-1,1]$ to $x \in[a, b]$. Then, when ${ }^{*} \mathcal{D}_{x} \equiv \partial^{1+v(x, t)} u / \partial|x|^{1+v(x, t)}$ in (17), the right-sided spatial differentiation matrix $\boldsymbol{D}_{\text {Riesz }}^{1+v}$ of Riesz type, corresponding to the nodal FLI expansion (33), is a three-dimensional matrix whose entries are given by

$$
\left\{\boldsymbol{D}_{\text {Riesz }}^{1+v(x, t)}\right\}_{i k m}=\left[\left(\frac{2}{b-a}\right)^{1+v} C_{1+v}\right]_{\left(x_{i}, t_{k}\right)} \sum_{j=1}^{\mathcal{M}} \widetilde{\beta}_{m j} \mathcal{W}_{j}^{\sigma}(x, t)
$$

in which $i, m=2,3, \cdots, \mathcal{M}, k=2,3, \cdots, \mathcal{N}, \xi=2 \frac{x-a}{b-a}$, and $\mathcal{W}_{j}^{\sigma}(x, t)$ is explicitly given as

$$
\begin{array}{r}
\mathcal{W}_{j}^{\nu}=\left(\frac{j+1}{2}\right) \sum_{q=\lceil\vee\rceil} C_{j} \frac{\left(\frac{-1}{2}\right)^{q} \Gamma(q+1)}{\Gamma(q+1-v(x, t))}\left[(-1)^{j-1}(1+\xi)^{q-v(x, t)}\right. \\
\left.-(1-\xi)^{q-v(x, t)}\right] .
\end{array}
$$

Proof. Given in Appendix F.

\subsection{Temporal Differentiation Matrix ${ }^{R L} \boldsymbol{D}_{t}^{\tau}$}

We derive the temporal differentiation matrix ${ }^{R L} \mathbf{D}_{t}^{\zeta}$ by taking ${ }_{0}^{R L} \mathcal{D}_{t}^{\zeta(x, t)} u_{N}(x, t)$ and evaluating it the collocation points considering the fact that the spatial nodal bases $L_{m}^{\mu}(x), R_{m}^{\mu}(x)$, and $h_{m}(x)$ all satisfy the Kronecker delta property at the collocation points. Hence, in the derivation of ${ }^{R L} \mathbf{D}_{t}^{\zeta}$ any of the expansions (28), (31), or (33) can be used. Following similar steps in section 5.1, we obtain the temporal differentiation matrix corresponding to the following two cases:

Case I-A) Constant $\zeta=\tau \in(0,1)$. We map the interval $t \in[0, T]$ to the standard domain $\xi \in[-1,1]$ as usual, and use the property (27) to obtain

$$
\left.{ }_{0}^{R L} \mathcal{D}_{t}^{\zeta} u_{N}(x, t)\right|_{\left(x_{i}, t_{k}\right)}=\sum_{n=2}^{\mathcal{N}}\left\{{ }^{R L} \mathbf{D}_{t}^{\zeta}\right\}_{k n} u_{N}\left(x_{i}, t_{n}\right)
$$


where $\left\{{ }^{R L} \mathbf{D}_{t}^{\zeta}\right\}_{\text {im }}$ are the entries of the $(\mathcal{N}-1) \times(\mathcal{N}-1)$ left-sided temporal differentiation matrix ${ }^{R L} \mathbf{D}_{L}^{\zeta}$ of Riemann-Liouville since, given by

$$
\left\{{ }^{R L} \mathbf{D}_{t}^{\zeta}\right\}_{k n}=\left(\frac{2}{T}\right)^{\zeta} \eta_{n} \sum_{j=1}^{\mathcal{N}} \beta_{n j}^{L} \frac{\Gamma(j+\zeta)}{\Gamma(j)} P_{j-1}\left(\xi_{k}\right)
$$

where $\eta_{n}=\frac{T}{2 t_{n}}$ and $\xi_{k}=\frac{2 t_{k}}{T}-1$.

Case I-B) The general $\zeta(x, t) \in(0,1)$. We obtain the corresponding temporal differentiation matrix of variable order $\zeta(x, t) \in(0,1)$, by evaluating ${ }_{0}^{R L} \mathcal{D}_{x}^{\zeta(x, t)} u_{N}(x, t)$ at the collocation points $\left(x_{i}, t_{k}\right)$ also by $L_{m}^{\mu}\left(x_{i}\right)=\delta_{i m}$, we obtain

$$
\begin{aligned}
\left.{ }_{0}^{R L} \mathcal{D}_{t}^{\zeta(x, t)} u_{N}\right|_{\left(x_{i}, t_{k}\right)} & =\left(\frac{2}{T}\right)^{\zeta\left(x_{i}, t_{k}\right)} \sum_{n=2}^{\mathcal{N}} u_{N}\left(x_{m}, t_{k}\right) \eta_{n} \sum_{j=1}^{\mathcal{N}} \beta_{n j}^{L} F_{j}^{L, \tau}\left(x_{i}, t_{k}\right) \\
& =\sum_{n=2}^{\mathcal{N}}\left\{{ }^{R L} \mathbf{D}_{t}^{\zeta}\right\}_{k n i} u_{N}\left(x_{i}, t_{n}\right),
\end{aligned}
$$

where $\left\{{ }^{R L} \mathbf{D}_{L}^{\zeta}\right\}_{k n i}$ are the entries of the $(\mathcal{N}-1) \times(\mathcal{M}-1) \times(\mathcal{N}-1)$ left-sided temporal fractional differentiation matrix ${ }^{R L} \mathbf{D}_{t}^{\zeta}$ of Riemann-Liouville sense, computed as

$$
\left\{{ }^{R L} \mathbf{D}_{t}^{\zeta}\right\}_{i k n}=\left(\frac{2}{T}\right)^{\zeta\left(x_{i}, t_{k}\right)} \eta_{n} \sum_{j=1}^{\mathcal{N}} \beta_{n j}^{L} F_{j}^{L, \tau}\left(x_{i}, t_{k}\right)
$$

We note that $\beta_{n j}^{L}$ are the corresponding coefficients in the following expansion that are obtained once as

$$
\mathcal{G}_{n}(\xi)=\sum_{j=1}^{\mathcal{N}} \beta_{n j}^{L} P_{j-1}^{-\tau, \tau}(\xi)
$$

similar to what we showed in (A.1).

\subsection{Temporal Differentiation Matrix ${ }^{C} D_{t}^{\tau}$}

We recall that when the time-derivative is of Caputo type we employ the expansion (35). 
Theorem 5.10. Let $\zeta=\zeta(x, t) \in C([a, b] \times[0, T])$ and consider the affine mapping that maps $\eta \in[-1,1]$ to $t \in[0, T]$. Then, when ${ }^{*} \mathcal{D}_{t} \equiv{ }_{0}^{C} \mathcal{D}_{t}$ in $(17)$, the leftsided temporal differentiation matrix ${ }^{C} \boldsymbol{D}^{\zeta}$ of Caputo type, corresponding to the nodal FLI expansion (35), is a three-dimensional matrix whose entries are given by

$$
\left\{{ }^{C} \boldsymbol{D}^{\zeta}\right\}_{i k m}=\left[\left(\frac{2}{T}\right)^{\zeta(x, t)}\right]_{\left(x_{i}, t_{k}\right)} \sum_{j=1}^{\mathcal{N}} \widetilde{\beta}_{m j} z_{j}^{\zeta}\left(x_{k}, t_{k}\right),
$$

when the solution to (17) is assumed to be continuous in time, in which $i, m=$ $2,3, \cdots, \mathcal{M}, k=1,2,3, \cdots, \mathcal{N}+1, \eta=\frac{2 t}{T}-1$, and $\widetilde{\beta}_{m j}$ are the corresponding (temporal) expansion coefficients, given a priori similarly as in (E.1). Moreover, $z_{j}^{\zeta}(x, t)$ is explicitly given as

$$
z_{j}^{\zeta}(x, t)=\frac{\Gamma(j+1)}{\Gamma\left(j-\zeta\left(x_{i}, t_{k}\right)+1\right)}\left[(1+\eta)^{-\zeta(x, t)} P_{j}^{\zeta,-\zeta}(\eta)\right] .
$$

Proof. See Appendix E.

\section{Numerical Tests}

After the construction of the variable-order differentiation matrices of RiemannLiouville and Riesz type, we now solve a number of FPDEs to investigate the performance of our schemes. We divide this section into two main parts. In the first part, we implement our variable-order collocation method in solving linear FPDEs such as time- and space-fractional advection equation, diffusion, and advection-diffusion problems. In the second part, we deal with non-linear FPDEs of field-variable order, namely the space-fractional nonlinear Burgers equation.

In all the numerical tests, we set collocation and interpolation points to be identical. Here, we adopt the choice of collocation points in [46], where we showed that the fractional extrema of ${ }^{(1)} \mathcal{P}_{n}^{\mu}(\xi)$ and ${ }^{(2)} \mathcal{P}_{n}^{\mu}(\xi)$, i.e., the zeros of Legendre polynomials, are the best collocation points leading to the fastest rate of convergence. To demonstrate the accuracy of our methods, we adopt the $L^{\infty}$ norm, normalized by the essential norm of the exact solution in each case.

\subsection{Linear FPDEs with ${ }^{*} \mathcal{D}_{x} \equiv{ }^{R L} \mathcal{D}_{x}$}

We first consider two examples of linear FPDEs, namely advection and advectiondiffusion problems, in which the spatial fractional derivatives are all either of leftor right-sided Riemann-Liouville type. 

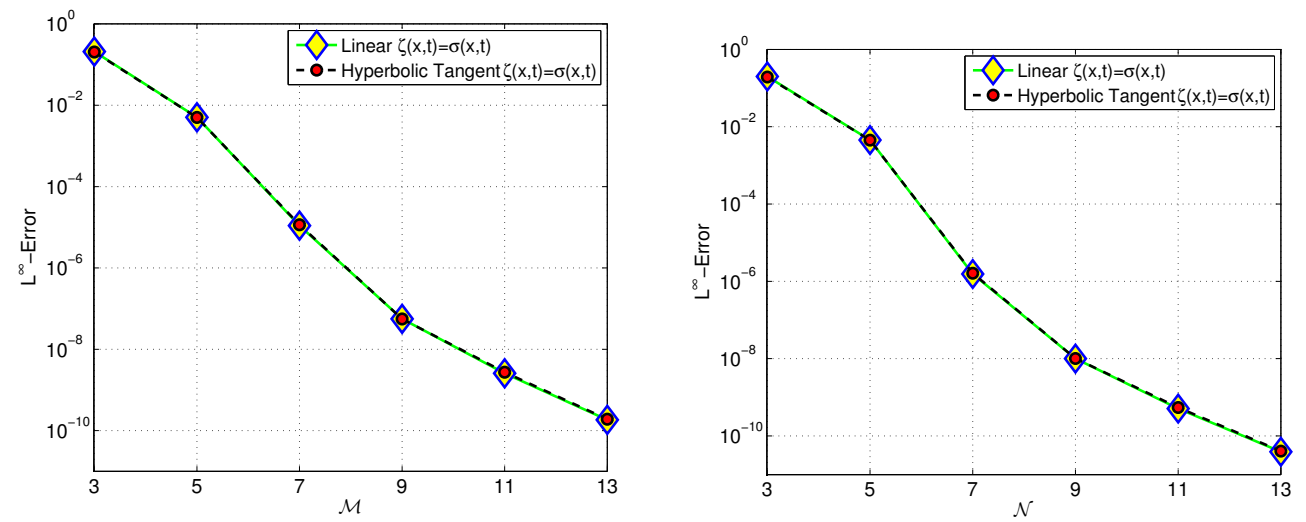

Figure 2: Time- and space-fractional linear advection problem with Riemann-Liouville spatial operators: (left) spatial $p$-refinement, and (right) temporal $p$-refinement. The exact solution is the fractional function $u^{e x t}(x, t)=(1+x)^{6+9 / 17} t^{6+2 / 3}$, where $(x, t) \in[-1,1] \times[0,2]$; moreover, the temporal and spatial fractional orders are taken as the following field-variable functions, denoted as linear $\zeta(x, t)=\sigma(x, t)=\left(\frac{5+4 x}{10}\right)\left(\frac{1+4 t}{10}\right)$, and hyperbolic tangent $\zeta(x, t)=\sigma(x, t)=[1+\tanh (x)][1+$ $\tanh (t-1)] / 4$.

Example 6.1. Fractional Advection Equation:

$$
\begin{aligned}
{ }_{0}^{R L} \mathcal{D}_{t}^{\zeta(x, t)} u+\theta^{R L} \mathcal{D}_{x}^{\sigma(x, t)} u & =f(x, t), \quad(x, t) \in[-1,1] \times[0,2], \\
u(x, 0) & =0 .
\end{aligned}
$$

where we replace $g(u)$ in (17) with the constant advection velocity $\theta$. Here, ${ }^{R L} \mathcal{D}_{x}^{\sigma} \equiv{ }_{-1}^{R L} \mathcal{D}_{x}^{\sigma}$ when $\theta>0$, hence we set the boundary condition $u(-1, t)=0$, and ${ }^{R L} \mathcal{D}_{x}^{\sigma} \equiv{ }_{x}{ }_{x} \mathcal{D}_{1}^{\sigma}$ when $\theta<0$, and therefore we set $u(1, t)=0$. Moreover in (58), $\zeta, \sigma: \mathbb{R}^{2} \rightarrow \mathbb{R}$ are continuous functions. In fact, we deduce the continuity requirement in $\zeta$ and $\sigma$ from the definition of the corresponding temporal and spatial differentiation matrices in (55) and (A.8), implying that each entry is assumed to be continuous from above and below. Let $\theta=1$ and seek the solution of the form (28), where we set the interpolation parameters $\tau$ and $\mu$ to be the mean-value of $\zeta(x, t)$ and $\sigma(x, t)$ given by

$$
\tau=\frac{1}{|\Omega|} \int_{\Omega} \zeta d \Omega
$$

and

$$
\mu=\frac{1}{|\Omega|} \int_{\Omega} \sigma d \Omega
$$


receptively. This is analogous to the algebraic mean-value interpolation parameters in multi-term FODEs/FPDEs, studied in [46]. We have investigated that such mean-values also lead to efficient approximation of fractional operators of variable-orders. Next, by substituting $u_{N}(x, t)$ in (58), we require the residual

$$
R_{N}(x, t)={ }_{0}^{R L} \mathcal{D}_{t}^{\zeta(x, t)} u_{N}+{ }_{-1}^{R L} \mathcal{D}_{x}^{\sigma(x, t)} u_{N}-f(x, t),
$$

to vanish at the collocation points $\left(x_{i}, t_{k}\right)$, which leads to the following linear system:

$$
R L \mathcal{S}_{A} \overrightarrow{\mathbf{u}}=\overrightarrow{\mathbf{b}},
$$

where ${ }^{R L} \mathcal{S}_{A}$ is a $(\mathcal{M}-1)(\mathcal{N}-1) \times(\mathcal{M}-1)(\mathcal{N}-1)$ matrix, whose entries are given by

$$
{ }^{R L} \mathcal{S}_{A}\left\{I_{i k}, J_{m n}\right\}=\left\{{ }^{R L} \mathbf{D}_{t}^{\zeta}\right\}_{i k n} \boldsymbol{\delta}_{i m}+\left\{{ }^{R L} \mathbf{D}_{L}^{\sigma}\right\}_{i k m} \boldsymbol{\delta}_{k n}
$$

where $I_{i k}=(i-2)(\mathcal{N}-1)+k-1, J_{m n}=(m-2)(\mathcal{N}-1)+n-1$, also $\left\{{ }^{R L} \mathbf{D}_{t}^{\zeta}\right\}_{i k n}$ and $\left\{{ }^{R L} \mathbf{D}_{L}^{\sigma}\right\}_{i k m}$ are given in (55) and (A.8), respectively. Moreover, $\overrightarrow{\mathbf{b}}$ represents the load vector whose components are obtained as

$$
\overrightarrow{\mathbf{b}}\left\{I_{i k}\right\}=f\left(x_{i}, t_{k}\right)
$$

and finally, the vector of unknown coefficients is defined as

$$
\overrightarrow{\mathbf{u}}\left\{J_{m n}\right\}=u\left(x_{m}, t_{n}\right),
$$

where $i, m=2,3, \cdots, \mathcal{M}$ and $k, n=2,3, \cdots, \mathcal{N}$.

In Fig. 2, we plot the exponential-like decay of $L^{\infty}$-norm of the error in corresponding spatial and temporal $p$-refinement. We set the exact solution to be the following fractional function $u^{\text {ext }}(x, t)=(1+x)^{6+9 / 17} t^{6+2 / 3}$. Moreover, the temporal and spatial fractional orders are taken as the following field-variable functions, denoted as linear $\zeta(x, t)=\sigma(x, t)=\left(\frac{5+4 x}{10}\right)\left(\frac{1+4 t}{10}\right)$, and hyperbolic tangent $\zeta(x, t)=\sigma(x, t)=[1+\tanh (x)][1+\tanh (t-1)] / 4$. We obtain identical results when we choose $\theta=-1$, i.e., the wind blows from right to left, and therefore right-sided Riemann-Liouville fractional derivatives are employed, considering $u^{\text {ext }}(x, t)=(1-x)^{6+9 / 17} t^{6+2 / 3}$. 

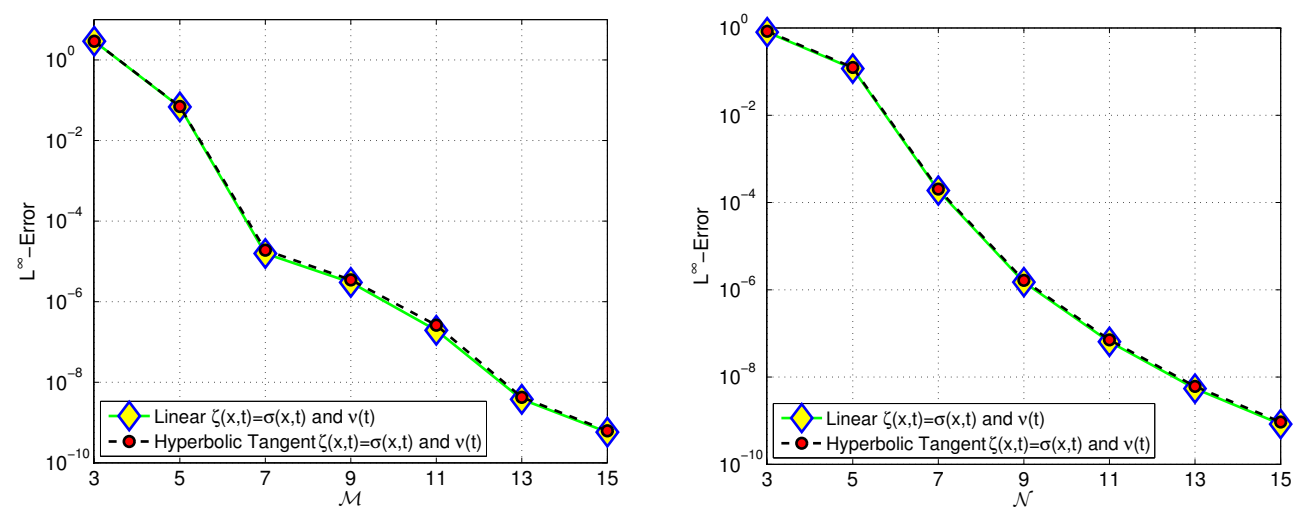

Figure 3: Time- and space-fractional linear advection-diffusion equation with Riemann-Liouville spatial operators: (left) spatial $p$-refinement, and (right) temporal $p$-refinement. The exact solution is given by the fractional $u^{e x t}(x, t)=(1+x)^{6+9 / 17} t^{6+2 / 3}$, where $(x, t) \in[-1,1] \times[0,2]$; moreover, the temporal and spatial fractional orders are respectively taken as the following field-variable functions, denoted as linear $\zeta(x, t)=\sigma(x, t)=\left(\frac{5+4 x}{10}\right)\left(\frac{1+4 t}{10}\right)$ and $1+v(t)=1+\frac{1+4 t}{10}$, also hyperbolic tangent $\zeta(x, t)=\sigma(x, t)=[1+\tanh (x)][1+\tanh (t-1)] / 4$ and $1+v(t)=[3+\tanh (t-1)] / 2$.

Example 6.2. Fractional Advection-Diffusion Equation:

$$
\begin{aligned}
{ }_{0}^{R L} \mathcal{D}_{t}^{\zeta(x, t)} u+\theta^{R L} \mathcal{D}_{x}^{\sigma(x, t)} u & ={ }^{R L} \mathcal{D}_{x}^{1+v(t)} u+f(x, t), \quad(x, t) \in[-1,1] \times[0,2] \\
u( \pm 1, t) & =0 \\
u(x, 0) & =0 .
\end{aligned}
$$

Here, ${ }^{R L} \mathcal{D}_{x}^{\sigma} \equiv{ }_{-1}^{R L} \mathcal{D}_{x}^{\sigma}$ and ${ }^{R L} \mathcal{D}_{x}^{1+v} u \equiv{ }_{-1}^{R L} \mathcal{D}_{x}^{1+v} u$ if $\theta>0$, i.e., when the wind blows from left to right. Alternatively, we set ${ }^{R L} \mathcal{D}_{x}^{\sigma} \equiv{ }_{x}^{R L} \mathcal{D}_{1}^{\sigma}$ and ${ }^{R L} \mathcal{D}_{x}^{1+v} u \equiv$ ${ }_{x}^{R L} \mathcal{D}_{1}^{1+v} u$ when $\theta<0$. We note that in (67), $\zeta: \mathbb{R}^{2} \rightarrow \mathbb{R}$ and $\sigma: \mathbb{R}^{2} \rightarrow \mathbb{R}$ are continuous functions. In addition, $v: \mathbb{R} \rightarrow \mathbb{R}$ suffices to be continuous in order for the entries of the differentiation matrices to be well-defined. We first set $\theta=1$ and seek the solution of the form (31), where we set the interpolation parameter $\mu$ to be the mean-value of $\sigma(x, t)$ given in (61). In a similar fashion, we can obtain the total average temporal interpolation parameter $\tau$ by including both $\zeta(x, t)$ and $v(t)$ into the averaging.

Having set the interpolation parameters, we substitute $u_{N}(x, t)$ in (67), and obtain the corresponding linear system by requiring the residual

$$
R_{N}(x, t)={ }_{0}^{R L} \mathcal{D}_{t}^{\zeta(x, t)} u_{N}+{ }_{-1}^{R L} \mathcal{D}_{x}^{\sigma(x, t)} u_{N}+{ }_{-1}^{R L} \mathcal{D}_{x}^{1+v(t)} u_{N}-f(x, t),
$$


to vanish at $\left(x_{i}, t_{k}\right)$. It then leads to

$$
{ }^{R L} \mathcal{S}_{A D} \overrightarrow{\mathbf{u}}=\overrightarrow{\mathbf{b}},
$$

where ${ }^{R L} \mathcal{S}_{A D}$ is a $(\mathcal{M}-2)(\mathcal{N}-1) \times(\mathcal{M}-2)(\mathcal{N}-1)$ matrix, whose entries are given by

$$
{ }^{R L} S_{A D}\left\{I_{i k}, J_{m n}\right\}=\left\{{ }^{R L} \mathbf{D}_{t}^{\zeta}\right\}_{i k n} \delta_{i m}+\left(\left\{{ }^{R L} \mathbf{D}_{L}^{\sigma}\right\}_{i k m}-\left\{{ }^{R L} \mathbf{D}_{L}^{1+v}\right\}_{i k m}\right) \delta_{k n}
$$

in which $I_{i k}=(i-2)(\mathcal{N}-1)+k-1, J_{m n}=(m-2)(\mathcal{N}-1)+n-1$, also $\left\{{ }^{R L} \mathbf{D}_{L}^{1+v}\right\}_{i k m}$ is given in (B.2), where $i, m=2,3, \cdots, \mathcal{M}-1$ and $k, n=2,3, \cdots, \mathcal{N}$.

In order to examine the temporal and spatial accuracy of our schemes for this model problem, we plot the $L^{\infty}$-error versus expansion order in each case in Fig. 3. We set the exact solution again as $u^{\text {ext }}(x, t)=(1+x)^{6+9 / 17} t^{6+2 / 3}$, where the temporal and spatial fractional orders are taken as the field-variable functions, denoted as linear and hyperbolic tangent. Once again, we observe the exponentiallike decays of error. We found identical convergence results in the case $\theta=-1$, where the corresponding spatial derivatives are of right-sided Riemann-Liouville type and $u^{\text {ext }}(x, t)=(1-x)^{6+9 / 17} t^{6+2 / 3}$.

\subsection{Linear FPDEs with Riesz Derivatives}

We consider two linear FPDEs with Riesz space-fractional derivatives of fieldvariable orders, namely as space- and time- fractional diffusion and advectiondiffusion problems.

Example 6.3. Fractional Diffusion Equation:

$$
\begin{aligned}
{ }_{0}^{R L} \mathcal{D}_{t}^{\zeta(x, t)} u & =\frac{\partial^{1+v(t)}}{\partial|x|^{1+v(t)}} u+f(x, t), \quad(x, t) \in[-1,1] \times[0, T] \\
u( \pm 1, t) & =0 \\
u(x, 0) & =0 .
\end{aligned}
$$

Here, we examine the same linear and hyperbolic tangent $\zeta(x, t)$ and $v(t)$ as in previous section. Hence, by setting $\tau=\mu=1 / 2$ as the average values for the aforementioned field-variable order, we seek the solution to (73) to be of the form (33). Next, we substitute $u_{N}(x, t)$ in (4), and require again the corresponding residual to vanish at $\left(x_{i}, t_{k}\right)$ to construct

$$
{ }^{\text {Riesz }} S_{D} \overrightarrow{\mathbf{u}}=\overrightarrow{\mathbf{b}}
$$



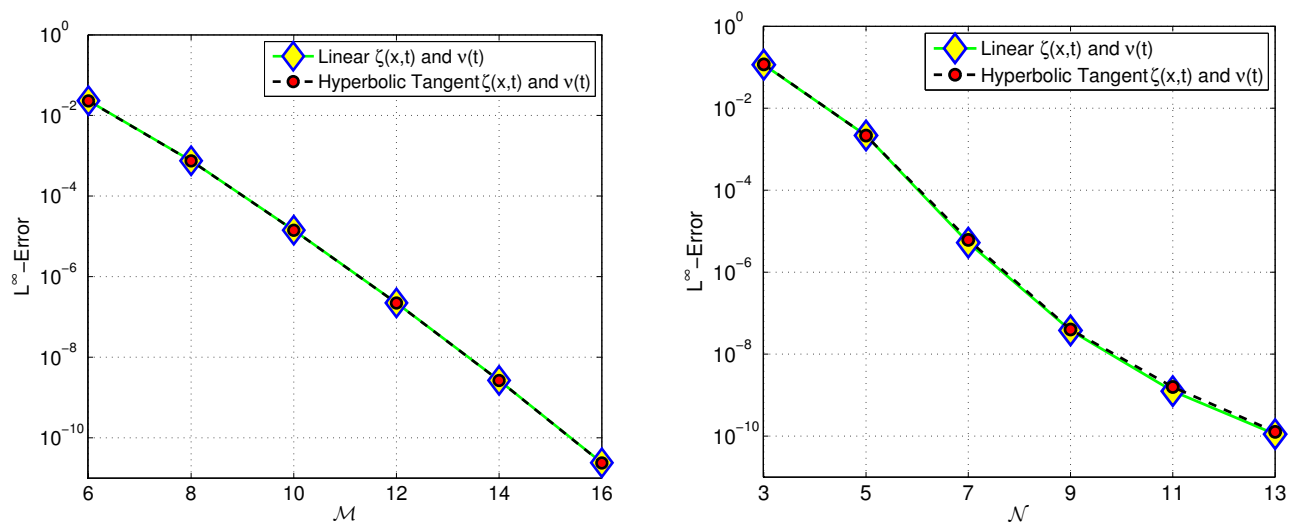

Figure 4: Time- and space-fractional linear diffusion equation with Riesz spatial operators: (left) spatial $p$-refinement, and (right) temporal $p$-refinement. The exact solution is given by the fractional $u^{e x t}(x, t)=\sin (\pi x) t^{6+2 / 3}$, where $(x, t) \in[-1,1] \times[0,2]$; moreover, the temporal and spatial fractional orders are respectively taken as the following field-variable functions, denoted as linear $\zeta(x, t)=\left(\frac{5+4 x}{10}\right)\left(\frac{1+4 t}{10}\right)$ and $1+\mathrm{v}(t)=1+\frac{1+4 t}{10}$, also hyperbolic tangent $\zeta(x, t)=$ $[1+\tanh (x)][1+\tanh (t-1)] / 4$ and $1+\mathrm{v}(t)=[3+\tanh (t-1)] / 2$.

where ${ }^{\text {Riesz }} \mathcal{S}_{D}$ is a $(\mathcal{M}-1)(\mathcal{N}-1) \times(\mathcal{M}-1)(\mathcal{N}-1)$ matrix, whose entries are given by

$$
{ }^{\text {Riesz }} \mathcal{S}_{D}\left\{I_{i k}, J_{m n}\right\}=\left\{{ }^{R L} \mathbf{D}_{t}^{\zeta}\right\}_{i k n} \boldsymbol{\delta}_{i m}-\left\{\mathbf{D}_{\text {Riesz }}^{1+v}\right\}_{i k m} \boldsymbol{\delta}_{k n},
$$

where $\left\{\mathbf{D}_{\text {Riesz }}^{1+v}\right\}_{i k m}$ is given in (F.5), in which $i, m=2,3, \cdots, \mathcal{M}$ and $k, n=2,3, \cdots, \mathcal{N}$. We note that in the nodal expansion (33), we consider $\mathcal{M}+1$, rather than $\mathcal{M}$ in the FLI (33), collocation points in the spatial dimension.

In a similar fashion, we plot the corresponding spatial and temporal $p$-refinement in Fig. 4, where we consider the exact solution this time to be $u^{\text {ext }}(x, t)=\sin (\pi x) t^{6+2 / 3}$, where we observe the exponential-like decay of the error versus the expansion order in each case.

Example 6.4. Fractional Advection-Diffusion Equation:

$$
\begin{aligned}
{ }_{0}^{R L} \mathcal{D}_{t}^{\zeta(x, t)} u+\frac{\partial^{\sigma(x, t)}}{\partial|x|^{\sigma(x, t)}} u & =\frac{\partial^{1+v(t)}}{\partial|x|^{1+v(t)}} u+f(x, t), \quad(x, t) \in[-1,1] \times[0,2] \\
u( \pm 1, t) & =0 \\
u(x, 0) & =0
\end{aligned}
$$



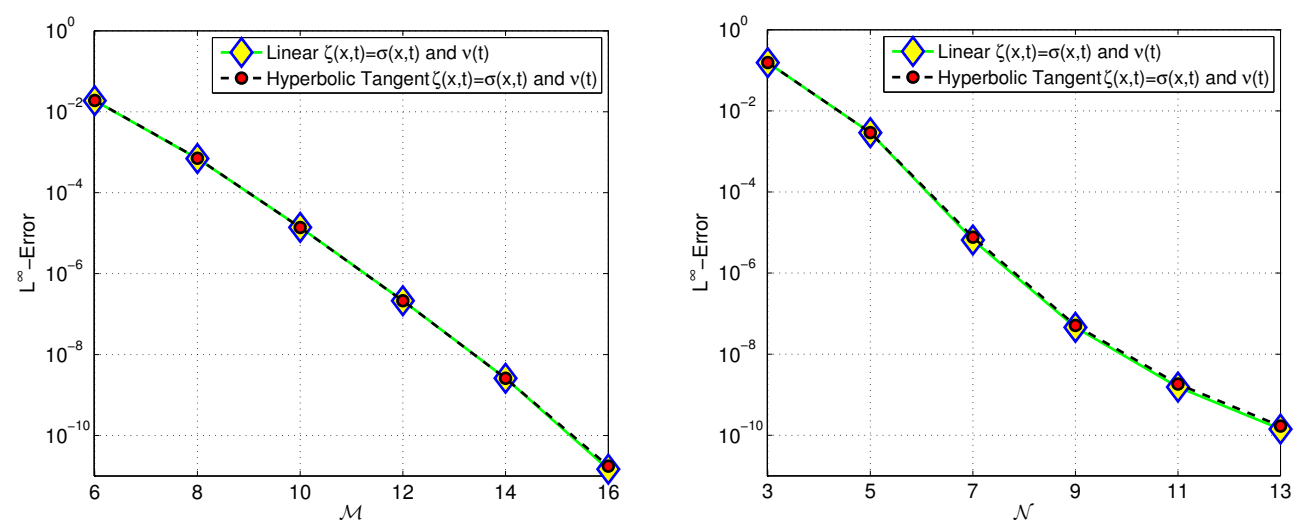

Figure 5: Time- and space-fractional linear advection-diffusion equation with Riesz spatial operators: (left) spatial $p$-refinement, and (right) temporal $p$-refinement. The exact solution is given by the fractional $u^{e x t}(x, t)=\sin (\pi x) t^{6+2 / 3}$, where $(x, t) \in[-1,1] \times[0,2]$; moreover, the temporal and spatial fractional orders are respectively taken as the following field-variable functions, denoted as linear $\zeta(x, t)=\sigma(x, t)=\left(\frac{5+4 x}{10}\right)\left(\frac{1+4 t}{10}\right)$ and $1+v(t)=1+\frac{1+4 t}{10}$, also hyperbolic tangent $\zeta(x, t)=\sigma(x, t)=[1+\tanh (x)][1+\tanh (t-1)] / 4$ and $1+v(t)=[3+\tanh (t-1)] / 2$.

We seek the solution to (78) to be of the form (33) and substitute it in (78). Then, we require the corresponding residual to vanish at $\left(x_{i}, t_{k}\right)$ to obtain

$$
{ }^{\text {Riesz }} \mathcal{S}_{A D} \overrightarrow{\mathbf{u}}=\overrightarrow{\mathbf{b}},
$$

where the entries of ${ }^{\text {Riesz }} \mathcal{S}_{A D}$ are given by

$$
{ }^{\text {Riesz }} \mathcal{S}_{A D}\left\{I_{i k}, J_{m n}\right\}=\left\{{ }^{R L} \mathbf{D}_{t}^{\zeta}\right\}_{i k n} \boldsymbol{\delta}_{i m}+\left(\left\{\mathbf{D}_{\text {Riesz }}^{\sigma}\right\}_{i k m}-\left\{\mathbf{D}_{\text {Riesz }}^{1+v}\right\}_{i k m}\right) \boldsymbol{\delta}_{k n},
$$

where $\left\{\mathbf{D}_{\text {Riesz }}^{\sigma}\right\}_{i k m}$ is given in (E.3), in which $i, m=2,3, \cdots, \mathcal{M}$ and $k, n=2,3, \cdots, \mathcal{N}$. Now, given the linear and hyperbolic tangent $\zeta(x, t)$ and $v(t)$ as in previous case, we set $\tau=\mu=1 / 2$ as the average values for the aforementioned field-variable order.

In Fig. 5, we present the exponential-like decay of $L^{\infty}$-error in the corresponding spatial and temporal $p$-refinement. The exact solution is again given by the fractional in-time function $u^{e x t}(x, t)=\sin (\pi x) t^{6+2 / 3}$. As before, the temporal and spatial fractional orders are respectively taken as the following field-variable functions, denoted as linear $\zeta(x, t)=\sigma(x, t)=\left(\frac{5+4 x}{10}\right)\left(\frac{1+4 t}{10}\right)$ and $1+\mathrm{v}(t)=1+\frac{1+4 t}{10}$, also hyperbolic tangent $\zeta(x, t)=\sigma(x, t)=[1+\tanh (x)][1+\tanh (t-1)] / 4$ and $1+\mathrm{v}(t)=[3+\tanh (t-1)] / 2$. 


\subsection{A Penalty Method for FPDEs}

We consider the following variable-order in time and space diffusion equation subject to the initial condition $u(x, 0)=g(x)$ and in absence of the any external forcing term.

\section{Example 6.5.}

$$
\begin{aligned}
{ }_{0}^{C} \mathcal{D}_{t}^{\zeta(x, t)} u & =\frac{\partial^{1+v(x, t)}}{\partial|x|^{1+v(x, t)}} u, \quad \forall(x, t) \in[-1,1] \times[0, T] \\
u( \pm 1, t) & =0 \\
u(x, 0) & =g(x),
\end{aligned}
$$

In this example, $u(x, t)$ is assumed to be continuous, and we employ the timederivative in (83) to be of Caputo type and the the spatial derivative of Riesz type. To enforce the inhomogeneous initial condition $u(x, 0)=g(x)$, we present the following penalty method as follows: find $u_{N}(x, t) \approx u(x, t)$ such that

$$
\begin{aligned}
{ }_{0}^{C} \mathcal{D}_{t}^{\zeta(x, t)} u_{N} & =\frac{\partial^{1+v(x, t)}}{\partial|x|^{1+v(x, t)}} u_{N}-\Xi Q^{-}(t)\left[u_{N}(x, 0)-g(x)\right], \\
u( \pm 1, t) & =0
\end{aligned}
$$

where $Q^{-}(0)=1$ and it vanishes at the rest of temporal collocation points $t \in$ $(0, T]$. Next, we seek the solution $u_{N}$ of the form

$$
u_{N}(x, t)=\sum_{m=2}^{\mathcal{M}} \sum_{n=1}^{\mathcal{N}+1} u_{N}\left(x_{m}, t_{n}\right) h_{m}(x) h_{n}(t),
$$

where $h_{n}(t)$ are the standard Legendre polynomials defined in $[0, T]$. This scheme is consistent since as $u_{N} \rightarrow u$ the penalty term vanishes asymptotically. Moreover, the global (spectral) treatment of the fractional time-derivative results in the penalty method in (86) to be unconditionally stable when $\Xi>0$, confirmed by our extensive numerical experiments. In fact, the bigger the penalty coefficient $\Xi$, the stronger enforcement of the initial condition is achieved. Hence, we set $\Xi=10^{15}$, which enforces the initial condition up to the machine precision. The dimension of the problem then becomes $(\mathcal{M}-1) \times(\mathcal{N}+1)$ since $u_{N}\left( \pm 1, t_{n}\right)=0$.

By substituting (88) in (83) and require the residual to vanish at the collocation points we obtain the following linear system

$$
{ }^{C R} \mathcal{S}_{D} \overrightarrow{\mathbf{u}}=\overrightarrow{\mathbf{b}}
$$



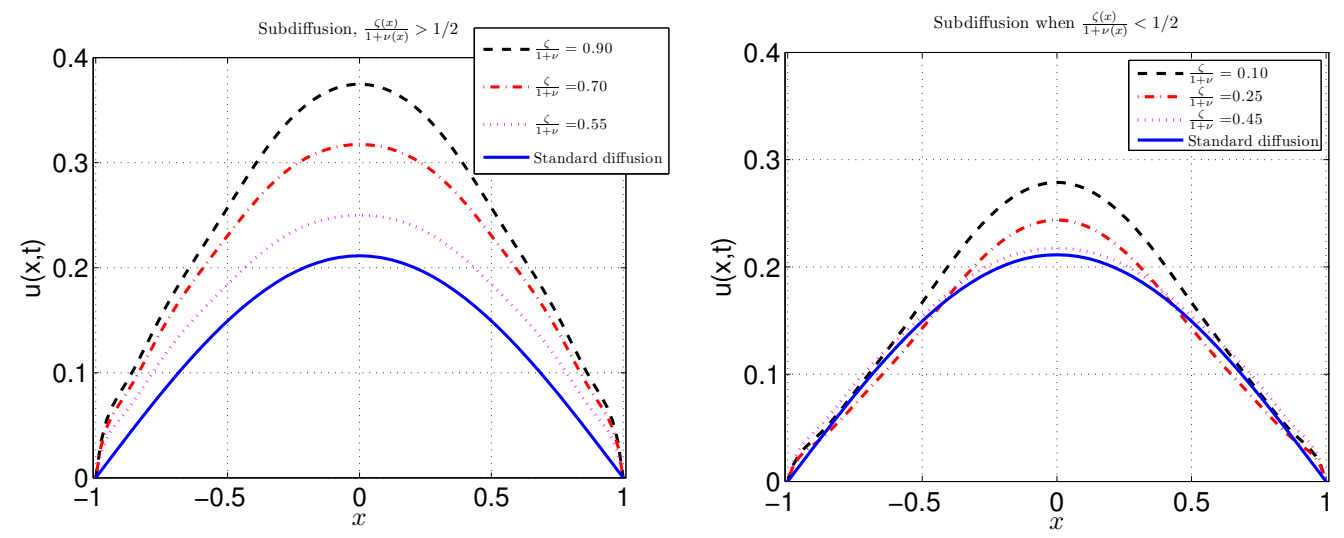

Figure 6: Variable-order diffusion problem: the initial condition is $u(x, 0)=\left(1-x^{2}\right)^{4}$ and the solutions are obtained at $t=\frac{1}{2}$, where the space-fractional order is $(1+v(x)) \in(1,2)$ and the time-fractional order is $\zeta(x) \in(0,1)$, defined as spatial functions, where the ratio $\zeta(x) /(1+v(x))$ is greater than $1 / 2$ (left), and is smaller than $1 / 2$ (right). In these test-cases, the fractional orders $\zeta(x)$ and $v(x)$ are given as constant when $x \in[-1 / 2,1 / 2]$ and they vary linearly towards the boundaries, such that they keep the ratio invariant.

in which

$$
{ }^{C R} S_{D}\left\{I_{i k}, J_{m n}\right\}=\left\{{ }^{C} \mathbf{D}_{t}^{\zeta}\right\}_{i k n} \delta_{i m}-\left\{\mathbf{D}_{\text {Riesz }}^{1+v}\right\}_{i k m} \delta_{k n}+\Xi \boldsymbol{\delta}_{m n} \boldsymbol{\delta}_{1 k}
$$

and

$$
\overrightarrow{\mathbf{b}}\left\{I_{i k}\right\}=-\Xi g\left(x_{i}\right) \boldsymbol{\delta}_{1 k},
$$

where $\left\{{ }^{C} \mathbf{D}_{t}^{\zeta}\right\}_{i k n}$ is given in (57), in which $i, m=2,3, \cdots, \mathcal{M}$ and $k, n=1,2, \cdots, \mathcal{N}+$ 1. Here, the prescript $C R$ recalls the Caputo and Riesz derivatives employed in the temporal and spatial dimensions, respectively.

In Fig. 6, we solve (86) subject to the initial condition is $u(x, 0)=\left(1-x^{2}\right)^{4}$ and we plot the results at $t=\frac{1}{2}$, where the space-fractional order is $(1+\mathrm{v}(x)) \in(1,2)$ and the time-fractional order is $\zeta(x) \in(0,1)$, defined as spatial functions, where the ratio $\zeta(x) /(1+v(x))$ is greater than $1 / 2$ (left), and is smaller than $1 / 2$ (right). Here, we keep the fractional orders $\zeta(x)$ and $v(x)$ constant when $x \in[-1 / 2,1 / 2]$ and allow them to decay linearly towards the boundaries, such that they keep the ratio invariant. The left plot reveals the sub-diffusion decay of the initial solution as expected. However, the right plot exhibits some local super-diffusive effects near the boundaries unexpectedly, which translates into faster decay compared to the standard diffusion. It is in contrast to the sub-diffusive nature of the problem 
when $\zeta \in(0,1)$. Such a local effect can be associated with the way we define $\zeta(x)$ and $v(x)$, which is only $x$-dependent when $x \in[-1,-1 / 2]$ and $x \in[1 / 2,1]$ and is constant in the middle domain. The polynomial order in time i.e., $\mathcal{N}$ and in space $\mathcal{M}$ are set to 18 is all simulations.

\subsection{Nonlinear FPDEs}

Here, we consider the nonlinear inviscid and viscous Burgers equation with field-variable orders in space.

Example 6.6. Inviscid and Viscous Burgers' Equation:

$$
\begin{aligned}
\frac{\partial u}{\partial t}+u^{*} \mathcal{D}_{x}^{\sigma(x, t)} u & =K^{*} \mathcal{D}_{x}^{1+v(x, t)} u+f(u ; x, t) \\
u( \pm 1, t) & =0 \\
u(x, 0) & =0
\end{aligned}
$$

Depending on the type of the fractional derivatives ${ }^{*} \mathcal{D}_{x}$, either of left-sided Riemann-Liouville or Riesz type, we seek the solution to (92) to be of the form

$$
u_{N}\left(x, t_{k}\right)=\sum_{m=2}^{\mathcal{M}-1} u_{N}\left(x_{m}, t_{k}\right) L_{m}^{\mu}(x)
$$

when ${ }^{*} \mathcal{D}_{x} \equiv{ }^{R L} \mathcal{D}_{x}$, given the homogeneous boundary conditions. Alternatively, we seek the solution to (92) to be of the form

$$
u_{N}\left(x, t_{k}\right)=\sum_{m=2}^{\mathcal{M}} u_{N}\left(x_{m}, t_{k}\right) h_{m}(x)
$$

when ${ }^{*} \mathcal{D}_{x}$ is of Riesz type. In this case, we employ a third-order Adams-Bashforth scheme to carry out the time-integration of (92), where we partition the time interval $[0, T]$ into equidistant points such that $\Delta t=T / N_{g}$, in which $N_{g}$ denotes the number of time-grid-points. Denoting by $\vec{u}_{N}^{j}$ the vector of solution at time $t_{j}=j \cdot \Delta t$, we obtain the following fully discrete form

$$
\frac{\vec{u}_{N}^{k+1}-\vec{u}_{N}^{k}}{\Delta t}=\sum_{q=1}^{g} \alpha_{q}\left(\left[-\operatorname{diag}\left(\vec{u}_{N}^{k+1-q}\right) \cdot \mathbf{D}^{\sigma}+K \mathbf{D}^{1+v}\right] \cdot \vec{u}_{N}^{k+1-q}+\vec{f}^{k+1-q}\right),
$$

where $\vec{f}^{k+1-q}=f\left(\vec{x}, t_{k+1-q}\right), \mathcal{J}$ represents the order of the method and $\alpha_{q}$ are the coefficients of the $\mathcal{I}$-th order Adams-Bashforth method. 
Table 1: $L^{\infty}$-norm error of the numerical solution to (92) with $\mathcal{M}$, corresponding to the fractional orders $\sigma(x, t)=v(x, t)=\left(\frac{5+4 x}{10}\right)\left(\frac{1+4 t}{10}\right)$, hence, we set $\mu=1 / 2$, the mean-value. The top table corresponds to the case where left-sided Riemann-Liouville fractional derivatives are employed. In this case, the exact solution is $u^{e x t}(x, t)=t^{3}(1+x)^{6+2 / 3}, x \in[-1,1]$ and we set $\Delta t=1 / 200$. The bottom table corresponds to the case where Riesz fractional derivatives are used and the exact solution is $u^{e x t}(x, t)=t^{3} \sin (\pi x), x \in[-1,1]$ and we set $\Delta t=1 / 600$. In both cases, the simulation time $T=1$, where in the third-order Adams-Bashforth time-integration scheme.

\begin{tabular}{|c|c|c|}
\hline \multicolumn{3}{|c|}{ Left-Sided Riemann-Liouville ${ }^{*} \mathcal{D}_{x} \equiv{ }^{R L} \mathcal{D}_{x}$} \\
\hline $\mathcal{M}$ & Inviscid Burgres $(K=0)$ & Viscous Burgres $(K=1)$ \\
\hline 5 & $1.88 \times 10^{-1}$ & $2.73 \times 10^{-1}$ \\
\hline 7 & $8.12 \times 10^{-4}$ & $8.82 \times 10^{-3}$ \\
\hline 9 & $1.44 \times 10^{-5}$ & $1.38 \times 10^{-5}$ \\
\hline \multicolumn{3}{|c|}{ Riesz Fractional Derivative ${ }^{*} \mathcal{D}_{x} \equiv \frac{\partial}{\partial|x|}$} \\
\hline $\mathcal{M}$ & Inviscid Burgres $(K=0)$ & Viscous Burgres $(K=1)$ \\
\hline 5 & $3.40 \times 10^{-2}$ & $1.42 \times 10^{-1}$ \\
\hline 7 & $3.93 \times 10^{-3}$ & $9.68 \times 10^{-3}$ \\
\hline 9 & $3.65 \times 10^{-4}$ & $6.32 \times 10^{-4}$ \\
\hline
\end{tabular}

Remark 6.7. The differentiation matrices $\mathbf{D}^{\sigma}$ and $\mathbf{D}^{1+v}$ are now two-dimensional matrices. However, we note that the entries of these matrices must be updated in each iteration due to the temporal variability in the fractional orders $\sigma(x, t)$ and $\mathrm{v}(x, t)$. To this end, we use (37) and (41) when ${ }^{*} \mathcal{D}_{x} \equiv{ }^{R L} \mathcal{D}_{x}$, where we substitute $t_{k}$ by $(k+1-q) \Delta t$. In addition, when ${ }^{*} \mathcal{D}_{x}$ is of Riesz type, we construct the twodimensional matrices $\mathbf{D}^{\sigma}$ and $\mathbf{D}^{1+v}$ using (52) and (53) similarly by substituting $t_{k}$ by $(k+1-q) \Delta t$ in each iteration.

In Table 1, we demonstrate the exponential decay of $L^{\infty}$-norm error of the numerical solution to (92) with $\mathcal{M}$, where left-sided Riemann-Liouville and Riesz fractional derivatives are employed. Here we examine field-variable fractional orders $\sigma(x, t)=\mathrm{v}(x, t)=\left(\frac{5+4 x}{10}\right)\left(\frac{1+4 t}{10}\right)$. When Riemann-Liouville derivatives are utilized, we set the exact solution to $u^{e x t}(x, t)=t^{3}(1+x)^{6+2 / 3}$ and $\Delta t=1 / 200$, and in the case of the Riesz fractional derivatives we set the exact solution $u^{\text {ext }}(x, t)=$ $t^{3} \sin (\pi x)$ while $\Delta t=1 / 600$. For both cases, the simulation time $T=1$ and we employ a third-order Adams-Bashforth time-integration scheme. 


\section{Summary and Discussion}

In this paper, we have developed a highly-accurate fractional spectral collocation method for solving linear and nonlinear FPDEs with field-variable temporal and/or spatial fractional orders. To this end and corresponding to the type of spatial derivatives (either Riemann-Liouville or Riesz), we introduced a new family of interpolants, called left-/right-sided and central fractional Lagrange interpolants, which satisfy the Kronecker delta property at collocation points. We constructed such interpolators to approximate the aforementioned fractional operators of both (left-/right-sided) Riemann-Liouville and Riesz type. We obtained the corresponding fractional differentiation matrices exactly. We solved several variable-order FPDEs including time- and space-fractional advection-equation, time- and space- fractional advection-diffusion equation, and finally the spacefractional Burgers' equation. We also developed an unconditionally stable penalty method that efficiently treats FPDEs subject to inhomogeneous initial conditions. In our numerical examples, we demonstrated the exponential decay of $L^{\infty}$-error in the aforementioned model problems.

In addition to the accuracy, the key idea to the efficiency in our approach was to collocate the fied-variable orders, as well as the resulting fractional FPDEs, at the collocation points. In fact, the $C^{0}$-continuity of the fractional order in fixed-order FDPEs allowed us to require only the $C^{0}$-continuity for the temporally and/or spatially-variable fractional orders in this study. This assumption made the pointwise evaluation of such field-variable orders at any arbitrary point well-defined in the space-time domain, hence, led us to circumvent the need for any quadrature rules that usually arise in spectral methods. To our experience, such numerical integrations become significantly costly when Galerkin/Petrov-Galerkin methods are employed, moreover, the resulting weak forms yield non-separable linear systems, which may lead to prohibitive computations in high dimensions.

\section{Appendix A. Proof of Theorem $5.1\left({ }^{*} \mathcal{D}_{x}^{\sigma(x, t)} \equiv{ }_{a}^{R L} \mathcal{D}_{x}^{\sigma(x, t)}\right)$}

We substitute (29) in (28) and take the $\sigma(x, t)$-th order fractional derivative. We do this by mapping the interval $x \in[a, b]$ to the standard domain $\xi \in[-1,1]$ 
through $x(\xi)=\frac{b+a}{2}+\frac{b-a}{2} \xi$ and following (10) as

$$
\begin{aligned}
{ }_{a}^{R L} \mathcal{D}_{x}^{\sigma(x, t)} u_{N} & =\left(\frac{2}{b-a}\right)^{\sigma(x(\xi), t)}{ }_{-1} \mathcal{D}_{\xi}^{\sigma(x(\xi), t)} u_{N}(x(\xi), t) \\
& =\left(\frac{2}{b-a}\right)^{\sigma}{ }_{-1}^{R L} \mathcal{D}_{\xi}^{\sigma}\left\{\sum_{m=2}^{\mathcal{M}} \sum_{n=1}^{\mathcal{N}} u_{N}\left(x_{m}, t_{n}\right) L_{m}^{\mu}(x(\xi)) \mathcal{T}_{n}^{\tau}(t)\right\} \\
& =\left(\frac{2}{b-a}\right)^{\sigma} \sum_{m=2}^{\mathcal{M}} \sum_{n=1}^{\mathcal{N}} u_{N}\left(x_{m}, t_{n}\right){ }_{-1}^{R L} \mathcal{D}_{\xi}^{\sigma}\left\{L_{m}^{\mu}(x(\xi))\right\} \mathcal{T}_{n}^{\tau}(t) \\
& =\left(\frac{2}{b-a}\right)^{\sigma} \sum_{m=2}^{\mathcal{M}} \sum_{n=1}^{\mathcal{N}} u_{N}\left(x_{m}, t_{n}\right){ }_{-1}^{R L} \mathcal{D}_{\xi}^{\sigma}\left\{\left(\frac{\xi-\xi_{1}}{\xi_{m}-\xi_{1}}\right)^{\mu} \prod_{k=1}^{\mathcal{M}}\left(\frac{\xi}{\xi_{k \neq m}-\xi_{k}}\right)\right\} \mathcal{T}_{n}^{\tau}(t) \\
& =\left(\frac{2}{b-a}\right)^{\sigma} \sum_{m=2}^{\mathcal{M}} \sum_{n=1}^{\mathcal{N}} u_{N}\left(x_{m}, t_{n}\right){ }_{-1}^{R L} \mathcal{D}_{\xi}^{\sigma}\left\{(1+\xi)^{\mu} \mathcal{G}_{m}(\xi)\right\} A_{m} \mathcal{T}_{n}^{\tau}(t)
\end{aligned}
$$

where $(2 /(b-a))^{\sigma(x, t)}$ is a strictly positive function, $A_{m}=1 /\left(\xi_{m}+1\right)^{\mu}$ and $\mathcal{G}_{m}(\xi)=$ $\prod_{\substack{k=1 \\ k \neq m}}^{\mathcal{M}}\left(\frac{\xi-\xi_{k}}{\xi_{m}-\xi_{k}}\right), m=2,3, \cdots, \mathcal{M}$, are all polynomials of order $(\mathcal{M}-1)$, which can be represented exactly in terms of Jacobi polynomials $P_{n-1}^{-\mu, \mu}(\xi)$ by

$$
\mathcal{G}_{m}(\xi)=\sum_{j=1}^{\mathcal{M}} \beta_{m j}^{L} P_{j-1}^{-\mu, \mu}(\xi) .
$$

We note that the unknown coefficient matrix $\beta_{m j}^{L}$ can be obtained analytically. The superscript ${ }^{L}$ actually refers to the proper change of basis to the regular eigenfunctions of FSLP (25) whose Left-sided fractional derivative is given exactly in (27). Next, by plugging (A.1) we obtain

$$
\begin{aligned}
{ }_{a}^{R L} \mathcal{D}_{x}^{\sigma(x, t)} u_{N} & =\left(\frac{2}{b-a}\right)^{\sigma} \sum_{m=2}^{\mathcal{M}} \sum_{n=1}^{\mathcal{N}} u_{N}\left(x_{m}, t_{n}\right){ }_{-1}^{R L} \mathcal{D}_{\xi}^{\sigma}\left\{(1+\xi)^{\mu} \sum_{j=1}^{\mathcal{M}} \beta_{m j}^{L} P_{j-1}^{-\mu, \mu}(\xi)\right\} A_{m} \mathcal{T}_{n}^{\tau}(t) \\
& =\left(\frac{2}{b-a}\right)^{\sigma} \sum_{m=2}^{\mathcal{M}} \sum_{n=1}^{\mathcal{N}} u_{N}\left(x_{m}, t_{n}\right) \sum_{j=1}^{\mathcal{M}} \beta_{m j-1}^{L}{ }_{-1}^{R L} \mathcal{D}_{\xi}^{\sigma}\left\{(1+\xi)^{\mu} P_{j-1}^{-\mu, \mu}(\xi)\right\} A_{m} \mathcal{T}_{n}^{\tau}(t)
\end{aligned}
$$

Hence, by (27) we obtain

$$
{ }_{a}^{R L} \mathcal{D}_{x}^{\sigma(x, t)} u_{N}=\left(\frac{2}{b-a}\right)^{\sigma} \sum_{m=2}^{\mathcal{M}} \sum_{n=1}^{\mathcal{N}} u_{N}\left(x_{m}, t_{n}\right) A_{m} \mathcal{T}_{n}^{\tau}(t) \sum_{j=1}^{\mathcal{M}} \beta_{m j-1}^{L}{ }^{R L} \mathcal{D}_{\xi}^{\sigma(1)} \mathcal{P}_{j}^{\mu}(\xi)
$$


Case A-I) Constant $\sigma=\mu \in(0,1)$. We use the property (27) and obtain

$$
{ }_{a}^{R L} \mathcal{D}_{x}^{\sigma} u_{N}=\left(\frac{2}{b-a}\right)^{\sigma} \sum_{m=2}^{\mathcal{M}} \sum_{n=1}^{\mathcal{N}} u_{N}\left(x_{m}, t_{n}\right) A_{m} \mathcal{T}_{n}^{\tau}(t) \sum_{j=1}^{\mathcal{M}} \beta_{m j}^{L} \frac{\Gamma(j+\sigma)}{\Gamma(j)} P_{j-1}(\xi) .
$$

Consequently, by evaluating ${ }_{a} \mathcal{D}_{x}^{\sigma} u_{N}(x, t)$ at the collocation points $\left(x_{i}, t_{k}\right)$ and recalling that $L_{n}^{\tau}\left(t_{k}\right)=\delta_{k n}$, we obtain

$$
\begin{aligned}
&\left.{ }_{a}^{R L} \mathcal{D}_{x}^{\sigma} u_{N}(x, t)\right|_{\left(x_{i}, t_{k}\right)}= \\
&\left(\frac{2}{b-a}\right)^{\sigma} \sum_{m=2}^{\mathcal{M}} \sum_{n=1}^{\mathcal{N}} u_{N}\left(x_{m}, t_{n}\right) A_{m} \delta_{k n} \sum_{j=1}^{\mathcal{M}} \beta_{m j}^{L} \frac{\Gamma(j+\sigma)}{\Gamma(j)} P_{j-1}\left(\xi_{i}\right) \\
&=\sum_{m=2}^{\mathcal{M}}\left\{{ }^{R L} \mathbf{D}_{L}^{\sigma}\right\}_{i m} u_{N}\left(x_{m}, t_{k}\right),
\end{aligned}
$$

where $\left\{{ }^{R L} \mathbf{D}_{L}^{\sigma}\right\}_{\text {im }}$ are the entries of the $(\mathcal{M}-1) \times(\mathcal{M}-1)$ left-sided spatial differentiation matrix ${ }^{R L} \mathbf{D}_{L}^{\sigma}$ of Riemann-Liouville type, given by

$$
\left\{{ }^{R L} \mathbf{D}_{L}^{\sigma}\right\}_{i m}=\left(\frac{2}{b-a}\right)^{\sigma} A_{m} \sum_{j=1}^{\mathcal{M}} \beta_{m j}^{L} \frac{\Gamma(j+\sigma)}{\Gamma(j)} P_{j-1}\left(\xi_{i}\right) .
$$

Case A-II) The general $\sigma(x, t) \in(0,1)$. Following [50] and [1], we first represent the polyfractonomial basis ${ }^{(2)} \mathcal{P}_{j}^{\mu}(\xi)$ in terms of a sum and take a left-sided Riemann-Liouville fractional derivative, to obtain $F_{j}^{L, \sigma}$ as

$$
\begin{aligned}
F_{j}^{L, \sigma}(x(\xi), t) & \equiv{ }_{-1}^{R L} \mathcal{D}_{\xi}^{\sigma}\left\{{ }^{(1)} \mathcal{P}_{j}^{\mu}(\xi)\right\} \\
& ={ }_{-1}^{R L} \mathcal{D}_{\xi}^{\sigma}\left\{\sum_{q=0}^{j-1}\left(\frac{1}{2}\right)^{q}(-1)^{q+j-1} b_{j q}^{\mu}(1+\xi)^{q+\mu}\right\} \\
& =\sum_{q=0}^{j-1}\left(\frac{1}{2}\right)^{q}(-1)^{q+j-1} b_{j q-1}^{*} \mathcal{D}_{\xi}^{\sigma}\left\{(1+\xi)^{q+\mu}\right\}
\end{aligned}
$$

where $b_{j q}^{*}=\left(\begin{array}{c}j-1+q \\ q\end{array}\right)\left(\begin{array}{c}j-1+\mu \\ j-1-q\end{array}\right)$. Now, we obtain $F_{j}^{L, \sigma}(x(\xi), t)$ exactly using (8) as

$$
F_{j}^{L, \sigma}(x(\xi), t)=\sum_{q=0}^{j-1} b_{j q}^{\mu}(1+\xi)^{q+\mu-\sigma}
$$


which leads to

$$
{ }_{a}^{R L} \mathcal{D}_{x}^{\sigma(x, t)} u_{N}(x, t)=\left(\frac{2}{b-a}\right)^{\sigma(x, t)} \sum_{m=2}^{\mathcal{M}} \sum_{n=1}^{\mathcal{N}} u_{N}\left(x_{m}, t_{n}\right) A_{m} \mathcal{T}_{n}^{\tau}(t) \sum_{j=1}^{\mathcal{M}} \beta_{m j}^{L} F_{j}^{L, \sigma}(x, t) .
$$

where

$$
b_{j q}^{\mu}=\left(\frac{1}{2}\right)^{q}(-1)^{q+j-1}\left(\begin{array}{c}
j-1+q \\
q
\end{array}\right)\left(\begin{array}{c}
j-1+\mu \\
j-1-q
\end{array}\right) \frac{\Gamma(q+\mu+1)}{\Gamma(q+\mu+1-\sigma)} .
$$

Now, by evaluating ${ }_{a}^{R L} \mathcal{D}_{x}^{\sigma(x, t)} u_{N}(x, t)$ at the collocation points $\left(x_{i}, t_{k}\right)$ also by $L_{n}^{\tau}\left(t_{k}\right)=$ $\delta_{k n}$, we obtain

$$
\begin{aligned}
\left.{ }_{a}^{R L} \mathcal{D}_{x}^{\sigma(x, t)} u_{N}\right|_{\left(x_{i}, t_{k}\right)} & =\left(\frac{2}{b-a}\right)^{\sigma\left(x_{i}, t_{k}\right)} \sum_{m=2}^{\mathcal{M}} u_{N}\left(x_{m}, t_{k}\right) A_{m} \sum_{j=1}^{\mathcal{M}} \beta_{m j}^{L} F_{j}^{L, \sigma}\left(x_{i}, t_{k}\right) \\
& =\sum_{m=2}^{\mathcal{M}}\left\{{ }^{R L} \mathbf{D}_{L}^{\sigma}\right\}_{i m k} u_{N}\left(x_{m}, t_{k}\right),
\end{aligned}
$$

where $\left\{{ }^{R L} \mathbf{D}_{L}^{\sigma}\right\}_{i k m}$ are the entries of the $(\mathcal{M}-1) \times(\mathcal{N}-1) \times(\mathcal{M}-1)$ left-sided spatial fractional differentiation matrix ${ }^{R L} \mathbf{D}_{L}^{\sigma}$ of Riemann-Liouville sense, computed as

$$
\left\{{ }^{R L} \mathbf{D}_{L}^{\sigma}\right\}_{i k m}=\left(\frac{2}{b-a}\right)^{\sigma\left(x_{i}, t_{k}\right)} A_{m} \sum_{j=1}^{\mathcal{M}} \beta_{m j}^{L} F_{j}^{L, \sigma}\left(x_{i}, t_{k}\right)
$$

where by re-arrangement $A_{m}=\left(\frac{b-a}{2 x_{m}-2 a}\right)^{\mu}$. It completes the proof.

Appendix B. Proof of Theorem $5.3\left({ }^{*} \mathcal{D}_{x}^{1+v(x, t)} \equiv{ }_{a}^{R L} \mathcal{D}_{x}^{1+v(x, t)}\right)$

We use the sequential property (6) and switch the order of $\partial / \partial x$ and ${ }_{a}^{R L} \mathcal{D}_{x}^{v(x, t)}$ to avoid the difficulties arising from taking the first partial derivative of the corresponding Euler gamma functions with respect to $x$. Hence,

$$
\begin{aligned}
& { }_{a}^{R L} \mathcal{D}_{x}^{1+v(x, t)} u_{N}(x, t)={ }_{a}^{R L} \mathcal{D}_{x}^{v(x, t)} \frac{\partial}{\partial x}\left\{u_{N}(x, t)\right\} \\
= & \left(\frac{2}{b-a}\right)^{v(x, t)}{ }_{-1}^{R L} \mathcal{D}_{\xi}^{v(x, t)}\left\{\sum_{m=2}^{\mathcal{M}} \sum_{n=1}^{\mathcal{N}} u_{N}\left(x_{m}, t_{n}\right) A_{m} \mathcal{T}_{n}^{\tau}(t) \sum_{j=1}^{\mathcal{M}} \beta_{m j}^{L}\left(\frac{2}{b-a}\right) \frac{\partial}{\partial \xi}\left[{ }^{(1)} \mathcal{P}_{j}^{\mu}(\xi)\right]\right\} \\
= & \left(\frac{2}{b-a}\right)^{(1+v(x, t))} \sum_{m=2}^{\mathcal{M}} \sum_{n=1}^{\mathcal{N}} u_{N}\left(x_{m}, t_{n}\right) A_{m} \mathcal{T}_{n}^{\tau}(t) \sum_{j=1}^{\mathcal{M}} \beta_{m j-1}^{L}{ }_{-1}^{R L} \mathcal{D}_{\xi}^{v(x, t)}\left\{\frac{\partial}{\partial \xi}\left[{ }^{(1)} \mathcal{P}_{j}^{\mu}(\xi)\right]\right\} .
\end{aligned}
$$


Now, by substituting (25) we obtain

$$
\begin{aligned}
{ }_{a}^{R L} \mathcal{D}_{x}^{1+v(x, t)} u_{N}(x, t) & =\left(\frac{2}{b-a}\right)^{(1+v(x, t))} \sum_{m=2}^{\mathcal{M}} \sum_{n=1}^{\mathcal{N}} u_{N}\left(x_{m}, t_{n}\right) A_{m} \mathcal{T}_{n}^{\tau}(t) . \\
\sum_{j=1}^{\mathcal{M}} \beta_{m j}^{L} & {\left[(\mu)_{-1}^{R L} \mathcal{D}_{\xi}^{v(x, t)}\left\{(1+\xi)^{\mu-1} P_{j-1}^{-\mu, \mu}(\xi)\right\}\right.} \\
& \left.+\left(\frac{j}{2}\right)_{-1}^{R L} \mathcal{D}_{\xi}^{v(x, t)}\left\{(1+\xi)^{\mu} P_{j-2}^{1-\mu, 1+\mu}(\xi)\right\}\right]
\end{aligned}
$$

in which the Jacobi polynomials $P_{j-1}^{-\mu, \mu}(\xi)$ and $P_{j-2}^{1-\mu, 1+\mu}(\xi)$ can be represented in terms of the following sums

$$
\begin{aligned}
P_{j-1}^{-\mu, \mu}(\xi) & =\sum_{q=0}^{j-1}\left(\frac{1}{2}\right)^{q}(-1)^{q+j-1} b_{j q}^{*}(1+\xi)^{q}, \quad j \geq 1 \\
P_{j-2}^{1-\mu, 1+\mu}(\xi) & =\sum_{q=0}^{j-2}\left(\frac{1}{2}\right)^{q}(-1)^{q+j-2} B_{j q}^{*}(1+\xi)^{q}, \quad j \geq 2
\end{aligned}
$$

respectively, where $B_{j q}^{*}=\left(\begin{array}{c}j+q \\ q\end{array}\right)\left(\begin{array}{c}j-1+\mu \\ j-2-q\end{array}\right)$. By substituting the Jacobi polynomials back into (B.1) and simplifying, it yields

$$
\begin{aligned}
{ }_{a}^{R L} \mathcal{D}_{x}^{1+v(x, t)} u_{N}(x, t) & =\left(\frac{2}{b-a}\right)^{1+v} \sum_{m=2}^{\mathcal{M}} \sum_{n=1}^{\mathcal{N}} u_{N}\left(x_{m}, t_{n}\right) A_{m} \mathcal{T}_{n}^{\tau}(t) . \\
\sum_{j=1}^{\mathcal{M}} \beta_{m j}^{L} & {\left[(\mu) \mathbb{I}_{\{j \geq 1\}} \sum_{q=0}^{j-1}\left(\frac{1}{2}\right)^{q}(-1)^{q+j-1} b_{j q-1}^{*}{ }^{R L} \mathcal{D}_{\xi}^{v(x, t)}\left\{(1+\xi)^{q+\mu-1}\right\}\right.} \\
& \left.+\left(\frac{j}{2}\right) \mathbb{I}_{\{j \geq 2\}} \sum_{q=0}^{j-2}\left(\frac{1}{2}\right)^{q}(-1)^{q+j-2} B_{j q-1}^{*}{ }^{R L} \mathcal{D}_{\xi}^{v(x, t)}\left\{(1+\xi)^{q+\mu}\right\}\right] .
\end{aligned}
$$


Now, by virtue of (8), we exactly obtain the variable-order fractional derivative of $u_{N}$ as

$$
\begin{aligned}
{ }_{a}^{R L} \mathcal{D}_{x}^{1+v(x, t)} u_{N}(x, t) & =\left(\frac{2}{b-a}\right)^{1+v} \sum_{m=2}^{\mathcal{M}} \sum_{n=1}^{\mathcal{N}} u_{N}\left(x_{m}, t_{n}\right) A_{m} \mathcal{T}_{n}^{\tau}(t) \cdot \\
\sum_{j=1}^{\mathcal{M}} \beta_{m j}^{L} & {\left[(\mu) \mathbb{I}_{\{j \geq 1\}} \sum_{q=0}^{j-1}\left(\frac{1}{2}\right)^{q}(-1)^{q+j-1} b_{j q}^{\mu} \frac{\Gamma(q+\mu)}{\Gamma(q+\mu-v(x, t))}(1+\xi)^{q+\mu-1-v}\right.} \\
& \left.+\left(\frac{j}{2}\right) \mathbb{I}_{\{j \geq 2\}} \sum_{q=0}^{j-2}\left(\frac{1}{2}\right)^{q}(-1)^{q+j-2} B_{j q}^{\mu} \frac{\Gamma(1+q+\mu)}{\Gamma(1+q+\mu-v(x, t))}(1+\xi)^{q+\mu-v}\right] .
\end{aligned}
$$

Next, by evaluating the above expression at the collocation points $\left(x_{i}, t_{k}\right)$, we obtain

$$
\left.a \mathcal{D}^{1+v(x, t)} u_{N}(x, t)\right|_{\left(x_{i}, t_{k}\right)}=\sum_{m=2}^{\mathcal{M}-1}\left\{{ }^{R L} \mathbf{D}_{L}^{1+v}\right\}_{i k m} u_{N}\left(x_{m}, t_{k}\right)
$$

in which $\left\{{ }^{R L} \mathbf{D}_{L}^{1+v}\right\}_{i k m}$, when $\boldsymbol{v}=\mathrm{v}(x, t)$ are computed as

$$
\left\{{ }^{R L} \mathbf{D}_{L}^{1+v}\right\}_{i k m}=\left(\frac{2}{b-a}\right)^{1+v\left(x_{i}, t_{k}\right)} A_{m} \sum_{j=1}^{\mathcal{M}} \beta_{m j}^{L} \mathcal{F}_{j}^{L, v}\left(x_{i}, t_{k}\right)
$$

where $i, m=2,3, \cdots, \mathcal{M}, k=2,3, \cdots, \mathcal{N}$, and $\mathcal{F}_{j}^{L, v}(x(\xi), t)$ is explicitly given by

$$
\begin{aligned}
\mathcal{F}_{j}^{L, \nu}(x(\xi), t) & =\mathbb{I}_{\{j \geq 1\}} \sum_{q=0}^{j-1} \mathbb{B}_{j q}^{\mu} \cdot(1+\xi)^{q+\mu-1-v\left(x_{i}, t_{k}\right)} \\
& +\mathbb{I}_{\{j \geq 2\}} \sum_{q=0}^{j-2} B_{j q}^{\mu} \cdot(1+\xi)^{q+\mu-v\left(x_{i}, t_{k}\right)}
\end{aligned}
$$

in which $j=1,2, \cdots, \mathcal{M}$, and $A_{m}=\left(\frac{b-a}{2 x_{m}-2 a}\right)^{\mu}$. Finally $\mathbb{B}_{j q}^{\mu}$ and $B_{j q}^{\mu}$ are the corresponding expansion coefficients, obtained as

$$
\mathbb{B}_{j q}^{\mu}=\mu\left(\frac{1}{2}\right)^{q}(-1)^{q+j-1}\left(\begin{array}{c}
j-1+q \\
q
\end{array}\right)\left(\begin{array}{l}
j-1+\mu \\
j-1-q
\end{array}\right) \frac{\Gamma(q+\mu)}{\Gamma\left(q+\mu-v\left(x_{i}, t_{k}\right)\right)},
$$


and

$$
B_{j q}^{\mu}=\frac{j}{2}\left(\frac{1}{2}\right)^{q}(-1)^{q+j-2}\left(\begin{array}{c}
j+q \\
q
\end{array}\right)\left(\begin{array}{c}
j-1+\mu \\
j-2-q
\end{array}\right) \frac{\Gamma(1+q+\mu)}{\Gamma\left(1+q+\mu-v\left(x_{i}, t_{k}\right)\right)} .
$$

\section{Appendix C. Proof of Theorem $5.5\left({ }^{*} \mathcal{D}_{x}^{\sigma(x, t)} \equiv{ }_{x}^{R L} \mathcal{D}_{b}^{\sigma(x, t)}\right)$}

We substitute (32) in (31) and take the $\sigma(x, t)$-th order fractional derivative as

$$
\begin{aligned}
{ }_{x}^{R L} \mathcal{D}_{b}^{\sigma(x, t)} u_{N} & =\left(\frac{2}{b-a}\right)^{\sigma(x(\xi), t)} \mathcal{D}_{1}^{\sigma(x(\xi), t)}\left\{u_{N}(x(\xi), t)\right\} \\
& =\left(\frac{2}{b-a}\right)^{\sigma} \sum_{m=2}^{\mathcal{M}} \sum_{n=1}^{\mathcal{N}} u_{N}\left(x_{m}, t_{n}\right){ }^{R L} \mathcal{D}_{1}^{\sigma}\left\{R_{m}^{\mu}(x(\xi))\right\} \mathcal{T}_{n}^{\tau}(t) \\
& =\left(\frac{2}{b-a}\right)^{\sigma} \sum_{m=2}^{\mathcal{M}} \sum_{n=1}^{\mathcal{N}} u_{N}\left(x_{m}, t_{n}\right){ }_{\xi^{R}}^{R} \mathcal{D}_{1}^{\sigma}\left\{\left(\frac{\xi_{\mathcal{M}}-\xi}{\xi_{\mathcal{M}}-\xi_{m}}\right)^{\mu} \prod_{k=1}^{\mathcal{M}}\left(\frac{\xi-\xi_{k}}{\xi_{m}-\xi_{k}}\right)\right\} \mathcal{T}_{n}^{\tau}(t) \\
& =\left(\frac{2}{b-a}\right)^{\sigma} \sum_{m=2}^{\mathcal{M}} \sum_{n=1}^{\mathcal{N}} u_{N}\left(x_{m}, t_{n}\right){ }_{-1}^{R L} \mathcal{D}_{\xi}^{\sigma}\left\{\left(1-\xi^{\mu} \mathcal{G}_{m}(\xi)\right\} \mathcal{A}_{m} \mathcal{T}_{n}^{\tau}(t)\right.
\end{aligned}
$$

in which $\mathcal{A}_{m}=1 /\left(\xi_{\mathcal{M}}-\xi_{m}\right)^{\mu}$ and this time we can re-represent $\mathcal{G}_{m}(\xi), m=$ $2,3, \cdots, \mathcal{M}$, in terms of another set of Jacobi polynomials $P_{n-1}^{\mu,-\mu}(\xi)$ exactly by

$$
\mathcal{G}_{m}(\xi)=\sum_{j=1}^{\mathcal{M}} \beta_{m j}^{R} P_{j-1}^{\mu,-\mu}(\xi)
$$

where the superscript ${ }^{R}$ in (C.1) now refers to the change of basis to the regular eigenfunctions of FSLP of second kind (26) whose Right-sided fractional derivative is given exactly in (27). We again highlight that the unknown coefficient matrix $\beta_{m j}^{R}$ can be obtained analytically. Next, by plugging (A.1) we obtain

$$
\begin{aligned}
{ }_{x}^{R L} \mathcal{D}_{b}^{\sigma(x, t)} u_{N} & =\left(\frac{2}{b-a}\right)^{\sigma} \sum_{m=2}^{\mathcal{M}} \sum_{n=1}^{\mathcal{N}} u_{N}\left(x_{m}, t_{n}\right){ }_{\xi}^{R L} \mathcal{D}_{1}^{\sigma}\left\{(1-\xi)^{\mu} \sum_{j=1}^{\mathcal{M}} \beta_{m j}^{R} P_{j-1}^{\mu,-\mu}(\xi)\right\} \mathcal{A}_{m} \mathcal{T}_{n}^{\tau}(t) \\
& =\left(\frac{2}{b-a}\right)^{\sigma} \sum_{m=2}^{\mathcal{M}} \sum_{n=1}^{\mathcal{N}} u_{N}\left(x_{m}, t_{n}\right) \sum_{j=1}^{\mathcal{M}} \beta_{m j}^{R}{ }_{\xi}^{R L} \mathcal{D}_{1}^{\sigma}\left\{(1-\xi)^{\mu} P_{j-1}^{\mu,-\mu}(\xi)\right\} \mathcal{A}_{m} \mathcal{T}_{n}^{\tau}(t)
\end{aligned}
$$


Hence, by (27)

$$
{ }_{x}^{R L} \mathcal{D}_{b}^{\sigma(x, t)} u_{N}=\left(\frac{2}{b-a}\right)^{\sigma} \sum_{m=2}^{\mathcal{M}} \sum_{n=1}^{\mathcal{N}} u_{N}\left(x_{m}, t_{n}\right) \mathcal{A}_{m} \mathcal{T}_{n}^{\tau}(t) \sum_{j=1}^{\mathcal{M}} \beta_{m j}^{R}{ }_{\xi}^{R L} \mathcal{D}_{1}^{\sigma}\left\{{ }^{(2)} \mathcal{P}_{j}^{\mu}(\xi)\right\}
$$

Case C-I) Constant $\sigma=\mu \in(0,1)$. By (27), we can directly obtain

$$
{ }_{x}^{R L} \mathcal{D}_{b}^{\sigma} u_{N}=\left(\frac{2}{b-a}\right)^{\sigma} \sum_{m=2}^{\mathcal{M}} \sum_{n=1}^{\mathcal{N}} u_{N}\left(x_{m}, t_{n}\right) \mathcal{A}_{m} \mathcal{T}_{n}^{\tau}(t) \sum_{j=1}^{\mathcal{M}} \beta_{m j}^{R} \frac{\Gamma(j+\sigma)}{\Gamma(j)} P_{j-1}(\xi),
$$

which we evaluate $\left(x_{i}, t_{k}\right)$ to obtain,

$$
\begin{aligned}
&\left.{ }_{x}^{R L} \mathcal{D}_{b}^{\sigma} u_{N}(x, t)\right|_{\left(x_{i}, t_{k}\right)}= \\
&\left(\frac{2}{b-a}\right)^{\sigma} \sum_{m=2}^{\mathcal{M}} \sum_{n=1}^{\mathcal{N}} u_{N}\left(x_{m}, t_{n}\right) \mathcal{A}_{m} \delta_{k n} \sum_{j=1}^{\mathcal{M}} \beta_{m j}^{R} \frac{\Gamma(j+\sigma)}{\Gamma(j)} P_{j-1}\left(\xi_{i}\right) \\
&=\sum_{m=2}^{\mathcal{M}}\left\{{ }^{R L} \mathbf{D}_{R}^{\sigma}\right\}_{i m} u_{N}\left(x_{m}, t_{k}\right),
\end{aligned}
$$

where $\left\{{ }^{R L} \mathbf{D}_{R}^{\sigma}\right\}_{\text {im }}$ are the entries of the $(\mathcal{M}-1) \times(\mathcal{M}-1)$ right-sided spatial differentiation matrix ${ }^{R L} \mathbf{D}_{R}^{\sigma}$ of Riemann-Liouville since, given by

$$
\left\{{ }^{R L} \mathbf{D}_{R}^{\sigma}\right\}_{i m}=\left(\frac{2}{b-a}\right)^{\sigma} \mathcal{A}_{m} \sum_{j=1}^{\mathcal{M}} \beta_{m j}^{R} \frac{\Gamma(j+\sigma)}{\Gamma(j)} P_{j-1}\left(\xi_{i}\right) .
$$

Case C-II) The general $\sigma(x, t) \in(0,1)$. Following [1], we first represent the polyfractonomial basis ${ }^{(2)} \mathcal{P}_{j}^{\mu}(\xi)$ in terms of the following sum then we take its right-sided Riemann-Liouville fractional derivative, denoted by $F_{j}^{R, \sigma}$, as

$$
\begin{aligned}
F_{j}^{R, \sigma}(x(\xi), t) & \equiv{ }_{\xi}^{R L} \mathcal{D}_{1}^{\sigma}\left\{{ }^{(2)} \mathcal{P}_{j}^{\mu}(\xi)\right\} \\
& ={ }_{\xi}^{R L} \mathcal{D}_{1}^{\sigma}\left\{\sum_{q=0}^{j-1}\left(\frac{-1}{2}\right)^{q} b_{j q}^{\mu}(1-\xi)^{q+\mu}\right\} \\
& =\sum_{q=0}^{j-1}\left(\frac{-1}{2}\right)^{q} c_{j q}^{*}{ }_{\xi}^{R L} \mathcal{D}_{1}^{\sigma}\left\{(1-\xi)^{q+\mu}\right\}
\end{aligned}
$$


where $c_{j q}^{*}=\left(\begin{array}{c}j-1+q \\ q\end{array}\right)\left(\begin{array}{c}j-1-\mu \\ j-1-q\end{array}\right)$. Now, we obtain $F_{j}^{R, \sigma}(x(\xi), t)$ exactly using (9) as

$$
F_{j}^{R, \sigma}(x(\xi), t)=\sum_{q=0}^{j-1} c_{j q}^{\mu}(1-\xi)^{q+\mu-\sigma}
$$

where

$$
c_{j q}^{\mu}=-\left(\frac{-1}{2}\right)^{q}\left(\begin{array}{c}
j-1+q \\
q
\end{array}\right)\left(\begin{array}{c}
j-1-\mu \\
j-1-q
\end{array}\right) \frac{\Gamma(q+\mu+1)}{\Gamma(q+\mu+1-\sigma)},
$$

which leads to

$$
{ }_{x}^{R L} \mathcal{D}_{b}^{\sigma(x, t)} u_{N}(x, t)=\left(\frac{2}{b-a}\right)^{\sigma(x, t)} \sum_{m=2}^{\mathcal{M}} \sum_{n=1}^{\mathcal{N}} u_{N}\left(x_{m}, t_{n}\right) \mathcal{A}_{m} \mathcal{T}_{n}^{\tau}(t) \sum_{j=1}^{\mathcal{M}} \beta_{m j}^{R} F_{j}^{R, \sigma}(x, t) .
$$

Now, by evaluating ${ }_{x}^{R L} \mathcal{D}_{b}^{\sigma(x, t)} u_{N}(x, t)$ at the collocation points $\left(x_{i}, t_{k}\right)$ we obtain

$$
\begin{aligned}
\left.{ }_{x}^{R L} \mathcal{D}_{b}^{\sigma(x, t)} u_{N}\right|_{\left(x_{i}, t_{k}\right)} & =\left(\frac{2}{b-a}\right)^{\sigma\left(x_{i}, t_{k}\right)} \sum_{m=2}^{\mathcal{M}} u_{N}\left(x_{m}, t_{k}\right) \mathcal{A}_{m} \sum_{j=1}^{\mathcal{M}} \beta_{m j}^{R} F_{j}^{R, \sigma}\left(x_{i}, t_{k}\right) \\
& =\sum_{m=2}^{\mathcal{M}}\left\{{ }^{R L} \mathbf{D}_{R}^{\sigma}\right\}_{i m k} u_{N}\left(x_{m}, t_{k}\right),
\end{aligned}
$$

where $\left\{{ }^{R L} \mathbf{D}_{R}^{\sigma}\right\}_{i k m}$ are the entries of the $(\mathcal{M}-1) \times(\mathcal{N}-1) \times(\mathcal{M}-1)$ right-sided spatial fractional differentiation matrix ${ }^{R L} \mathbf{D}_{R}^{\sigma}$ of Riemann-Liouville sense, computed as

$$
\left\{{ }^{R L} \mathbf{D}_{R}^{\sigma}\right\}_{i k m}=\left(\frac{2}{b-a}\right)^{\sigma\left(x_{i}, t_{k}\right)} \mathcal{A}_{m} \sum_{j=1}^{\mathcal{M}} \beta_{m j}^{R} F_{j}^{R, \sigma}\left(x_{i}, t_{k}\right)
$$

where by re-arrangement $\mathcal{A}_{m}=\left(\frac{b-a}{2 b-2 x_{m}}\right)^{\mu}$. We recall from Remark 5.2 that if $\sigma=\sigma(x)$, we again reduce the dimension of ${ }^{R L} \mathbf{D}_{R}^{\sigma}$ by one, hence, we can obtain the entries of the corresponding two-dimensional right-sided differentiation matrix as

$$
\left\{{ }^{R L} \mathbf{D}_{R}^{\sigma}\right\}_{i m}=\left(\frac{2}{b-a}\right)^{\sigma\left(x_{i}\right)} \mathcal{A}_{m} \sum_{j=1}^{\mathcal{M}} \beta_{m j}^{L} F_{j}^{R, \sigma}\left(x_{i}\right) .
$$


Appendix D. Proof of Theorem $5.6\left({ }^{*} \mathcal{D}_{x}^{\sigma(x, t)} \equiv{ }_{x}^{R L} \mathcal{D}_{b}^{1+v(x, t)}\right)$

When the fractional order is both $x$-and $t$-dependent, to avoid the difficulties in computations of the first partial derivative in the corresponding gamma functions with respect to $x$, we alternatively write ${ }_{x}^{R L} \mathcal{D}_{b}^{1+v(x, t)}$ as

$$
\begin{aligned}
& { }_{x}^{R L} \mathcal{D}_{b}^{1+v(x, t)} u_{N}(x, t)={ }_{x}^{R L} \mathcal{D}_{b}^{v(x, t)} \frac{\partial}{\partial x}\left\{u_{N}(x, t)\right\} \\
& =\left(\frac{2}{b-a}\right)^{v}{ }_{\xi}^{R L} \mathcal{D}_{1}^{v(x, t)}\left\{\sum_{m=2}^{\mathcal{M}} \sum_{n=1}^{\mathcal{N}} u_{N}\left(x_{m}, t_{n}\right) \mathcal{A}_{m} \mathcal{T}_{n}^{\tau}(t) \sum_{j=1}^{\mathcal{M}} \beta_{m j}^{L}\left(\frac{2}{b-a}\right) \frac{\partial}{\partial \xi}\left[{ }^{(2)} \mathcal{P}_{j}^{\mu}(\xi)\right]\right\} \\
& \quad=\left(\frac{2}{b-a}\right)^{1+v} \sum_{m=2}^{\mathcal{M}} \sum_{n=1}^{\mathcal{N}} u_{N}\left(x_{m}, t_{n}\right) \mathcal{A}_{m} \mathcal{T}_{n}^{\tau}(t) \sum_{j=1}^{\mathcal{M}} \beta_{m j}^{L}{ }_{\xi}^{R L} \mathcal{D}_{1}^{v(x, t)}\left\{\frac{\partial}{\partial \xi}\left[{ }^{(2)} \mathcal{P}_{j}^{\mu}(\xi)\right]\right\}
\end{aligned}
$$

Now, by substituting (26) we obtain

$$
\begin{aligned}
{ }_{x}^{R L} \mathcal{D}_{b}^{1+v(x, t)} u_{N}(x, t) & =\left(\frac{2}{b-a}\right)^{1+v} \sum_{m=2}^{\mathcal{M}} \sum_{n=1}^{\mathcal{N}} u_{N}\left(x_{m}, t_{n}\right) \mathcal{A}_{m} \mathcal{T}_{n}^{\tau}(t) \\
\sum_{j=1}^{\mathcal{M}} \beta_{m j}^{L} & {\left[(-\mu)^{R L} \mathcal{D}_{1}^{v(x, t)}\left\{(1-\xi)^{\mu-1} P_{j-1}^{\mu,-\mu}(\xi)\right\}\right.} \\
& \left.+\left(\frac{j}{2}\right)^{R L}{ }_{\xi}^{R} \mathcal{D}_{1}^{v(x, t)}\left\{(1-\xi)^{\mu} P_{j-2}^{1+\mu, 1-\mu}(\xi)\right\}\right]
\end{aligned}
$$

in which the Jacobi polynomials $P_{j-1}^{\mu,-\mu}(\xi)$ and $P_{j-2}^{1+\mu, 1-\mu}(\xi)$ can be represented in terms of the following sums

$$
\begin{aligned}
P_{j-1}^{\mu,-\mu}(\xi) & =\sum_{q=0}^{j-1}\left(\frac{-1}{2}\right)^{q} c_{j q}^{\mu} \cdot(1-\xi)^{q}, \quad j \geq 1, \\
P_{j-2}^{1+\mu, 1-\mu}(\xi) & =\sum_{q=0}^{j-2}\left(\frac{-1}{2}\right)^{q} B_{j q}^{\mu} \cdot(1-\xi)^{q}, \quad j \geq 2,
\end{aligned}
$$


respectively. Now, by substituting the Jacobi polynomials back into (D.1) and simplifying, it yields

$$
\begin{aligned}
{ }_{x}^{R L} \mathcal{D}_{b}^{1+v(x, t)} u_{N}(x, t) & =\left(\frac{2}{b-a}\right)^{1+v} \sum_{m=2}^{\mathcal{M}} \sum_{n=1}^{\mathcal{N}} u_{N}\left(x_{m}, t_{n}\right) \mathcal{A}_{m} \mathcal{T}_{n}^{\tau}(t) \cdot \\
\sum_{j=1}^{\mathcal{M}} \beta_{m j}^{L} & {\left[(-\mu) \mathbb{I}_{\{j \geq 1\}} \sum_{q=0}^{j-1}\left(\frac{-1}{2}\right)^{q} c_{j q}^{*}{ }_{\xi}^{R L} \mathcal{D}_{1}^{v(x, t)}\left\{(1-\xi)^{q+\mu-1}\right\}\right.} \\
& \left.+\left(\frac{j}{2}\right) \mathbb{I}_{\{j \geq 2\}} \sum_{q=0}^{j-2}\left(\frac{-1}{2}\right)^{q} C_{j q}^{*}{ }_{\xi}^{R L} \mathcal{D}_{1}^{v(x, t)}\left\{(1-\xi)^{q+\mu}\right\}\right]
\end{aligned}
$$

where $C_{j q}^{*}=\left(\begin{array}{c}j+q \\ q\end{array}\right)\left(\begin{array}{c}j-1+\mu \\ j-2-q\end{array}\right)$. Now, by virtue of (9), we exactly obtain the variable-order fractional derivative of $u_{N}$ as

$$
\begin{aligned}
{ }_{x}^{R L} \mathcal{D}_{b}^{1+v(x, t)} u_{N}(x, t) & =\left(\frac{2}{b-a}\right)^{1+v} \sum_{m=2}^{\mathcal{M}} \sum_{n=1}^{\mathcal{N}} u_{N}\left(x_{m}, t_{n}\right) \mathcal{A}_{m} \mathcal{T}_{n}^{\tau}(t) \\
\sum_{j=1}^{\mathcal{M}} \beta_{m j}^{L} & {\left[\mathbb{I}_{\{j \geq 1\}} \sum_{q=0}^{j-1} \mathbb{C}_{j q}^{\mu}(1-\xi)^{q+\mu-1-v}\right.} \\
& \left.+\mathbb{I}_{\{j \geq 2\}} \sum_{q=0}^{j-2} C_{j q}^{\mu}(1-\xi)^{q+\mu-v}\right] .
\end{aligned}
$$

where

$$
\mathbb{C}_{j q}^{\mu}=(\mu)\left(\frac{-1}{2}\right)^{q}\left(\begin{array}{c}
j-1+q \\
q
\end{array}\right)\left(\begin{array}{c}
j-1-\mu \\
j-1-q
\end{array}\right) \frac{\Gamma(q+\mu)}{\Gamma(q+\mu-v(x, t))}
$$

and

$$
C_{j q}^{\mu}=\left(\frac{-j}{2}\right)\left(\frac{-1}{2}\right)^{q}\left(\begin{array}{c}
j+q \\
q
\end{array}\right)\left(\begin{array}{c}
j-1+\mu \\
j-2-q
\end{array}\right) \frac{\Gamma(1+q+\mu)}{\Gamma(1+q+\mu-v(x, t))} .
$$

Next, by evaluating the above expression at the collocation points $\left(x_{i}, t_{k}\right)$, we obtain

$$
\left\{{ }^{R L} \mathbf{D}_{R}^{1+v}\right\}_{i k m}=\left(\frac{2}{b-a}\right)^{1+v\left(x_{i}, t_{k}\right)} \mathcal{A}_{m} \sum_{j=1}^{\mathcal{M}} \beta_{m j}^{R} \mathcal{F}_{j}^{R, v}\left(x_{i}, t_{k}\right)
$$


where $\mathcal{F}_{j}^{R, v}(x(\xi), t)$ is obtained as

$$
\begin{aligned}
\mathcal{F}_{j}^{R, v}(x(\xi), t) & =\mathbb{I}_{\{j \geq 1\}} \sum_{q=0}^{j-1} \mathbb{C}_{j q}^{\mu} \cdot(1-\xi)^{q+\mu-1-v\left(x_{i}, t_{k}\right)} \\
& +\mathbb{I}_{\{j \geq 2\}} \sum_{q=0}^{j-2} C_{j q}^{\mu} \cdot(1-\xi)^{q+\mu-v\left(x_{i}, t_{k}\right)}
\end{aligned}
$$

which completes the proof.

\section{Appendix E. Proof of Theorem $5.8\left({ }^{*} \mathcal{D}_{x}^{\sigma(x, t)} \equiv \partial^{\sigma(x, t)} u / \partial|x|^{\sigma(x, t)}\right)$}

We substitute (34) in (33) and take the $\sigma(x, t)$-th order fractional derivative. Once again, we perform the affine mapping from $x \in[a, b]$ to $\xi \in[-1,1]$ as before an we obtain

$$
\begin{gathered}
\frac{\partial^{\sigma(x, t)} u_{N}(x, t)}{\partial|x|^{\sigma(x, t)}}=\left(\frac{2}{b-a}\right)^{\sigma(x(\xi), t)} C_{\sigma(x(\xi), t)}\left({ }_{-1} \mathcal{D}_{\xi}^{\sigma} u_{N}+{ }_{\xi} \mathcal{D}_{1}^{\sigma} u_{N}\right) \\
=\left(\frac{2}{b-a}\right)^{\sigma} C_{\sigma} \sum_{m=2}^{\mathcal{M}} \sum_{n=1}^{\mathcal{N}} u_{N}\left(x_{m}, t_{n}\right)\left({ }_{-1}^{R L} \mathcal{D}_{\xi}^{\sigma}\left\{h_{m}(x(\xi))\right\}+{ }^{R L} \mathcal{D}_{1}^{\sigma}\left\{h_{m}(x(\xi))\right\}\right) \mathcal{T}_{n}^{\tau}(t),
\end{gathered}
$$

where $h_{m}(x(\xi)) \equiv h_{m}(\xi)$ are all polynomials of order $(\mathcal{M})$, which can be represented exactly in terms of Legendre polynomials $P_{n}(\xi)$ as

$$
h_{m}(\xi)=\sum_{j=0}^{\mathcal{M}} \widetilde{\beta}_{m j} P_{j}(\xi) .
$$

We again note that the coefficient matrix $\widetilde{\beta}_{m j}$ can be obtained analytically. By plugging (E.1) we obtain

$$
\begin{aligned}
& \frac{\partial^{\sigma(x, t)} u_{N}(x, t)}{\partial|x|^{\sigma(x, t)}}= \\
& \left(\frac{2}{b-a}\right)^{\sigma} C_{\sigma} \sum_{m=2}^{\mathcal{M}} \sum_{n=1}^{\mathcal{N}} u_{N}\left(x_{m}, t_{n}\right) \sum_{j=0}^{\mathcal{M}} \widetilde{\beta}_{m j}\left({ }_{-1}^{R L} \mathcal{D}_{\xi}^{\sigma}\left\{P_{j}(\xi)\right\}+{ }_{\xi}^{R L} \mathcal{D}_{1}^{\sigma}\left\{P_{j}(\xi)\right\}\right) \mathcal{T}_{n}^{\tau}(t),
\end{aligned}
$$


where by exact evaluation of the left- and right-sided fractional derivatives of the Legendre polynomials in (50) and (51),

$$
\begin{gathered}
\frac{\partial^{\sigma(x, t)} u_{N}(x, t)}{\partial|x| \sigma(x, t)}=\left(\frac{2}{b-a}\right)^{\sigma} C_{\sigma} \sum_{m=2}^{\mathcal{M}} \sum_{n=1}^{\mathcal{N}} u_{N}\left(x_{m}, t_{n}\right)\{ \\
\left.\left.\sum_{j=\lceil\sigma(x, t)\rceil}^{\mathcal{M}} \widetilde{\beta}_{m j} \frac{\Gamma(j+1)}{\Gamma(j-\sigma+1)}\left\{(1+\xi)^{-\sigma} P_{j}^{\sigma,-\sigma}(\xi)+(1-\xi)^{-\sigma} P_{j}^{-\sigma, \sigma}(\xi)\right\}\right\} \mathcal{T}_{n}^{\tau}(t)\right\}(\mathrm{E}, 2)
\end{gathered}
$$

which we evaluate at the collocation points $u_{N}\left(x_{i}, t_{k}\right)$ to obtain

$$
\left.\frac{\partial^{\sigma(x, t)} u_{N}(x, t)}{\partial|x|^{\sigma(x, t)}}\right|_{\left(x_{i}, t_{k}\right)}=\sum_{m=2}^{\mathcal{M}}\left\{\mathbf{D}_{\text {Riesz }}^{\sigma}\right\}_{i k m} u_{N}\left(x_{m}, t_{k}\right)
$$

in which $\left\{\mathbf{D}_{\text {Riesz }}^{\sigma}\right\}_{i k m}$ are the entries of the three dimensional Riesz spatial differentiation matrix of order $\sigma\left(x_{i}, t_{k}\right), \mathbf{D}_{\text {Riesz }}^{\sigma}$, given by

$$
\left\{\mathbf{D}_{\text {Riesz }}^{\sigma}\right\}_{i k m}=\left[\left(\frac{2}{b-a}\right)^{\sigma(x, t)} C_{\sigma(x, t)}\right]_{\left(x_{i}, t_{k}\right)} \sum_{j=1}^{\mathcal{M}} \widetilde{\beta}_{m j} z_{j}^{\sigma}\left(x_{k}, t_{k}\right)
$$

where

$$
Z_{j}^{\sigma}(x, t)=\frac{\Gamma(j+1)}{\Gamma\left(j-\sigma\left(x_{i}, t_{k}\right)+1\right)}\left[(1+\xi)^{-\sigma(x, t)} P_{j}^{\sigma,-\sigma}(\xi)+(1-\xi)^{-\sigma(x, t)} P_{j}^{-\sigma, \sigma}(\xi)\right],
$$

in which we recall that $\xi=2 \frac{x-a}{b-a}$.

Appendix F. Proof of Theorem 5.9 $\left({ }^{*} \mathcal{D}_{x}^{1+v(x, t)} \equiv \partial^{1+v(x, t)} u / \partial|x|^{1+v(x, t)}\right)$

We first take the fractional and then the first derivative of the solution to obtain

$$
\begin{gathered}
\frac{\partial^{1+v(x, t)} u_{N}(x, t)}{\partial|x|^{1+v(x, t)}}=C_{1+v}\left\{{ }_{a}^{R L} \mathcal{D}_{x}^{v(x, t)}+{ }^{R L} \mathcal{D}_{b}^{v(x, t)}\right\}\left(\frac{\partial u_{N}}{\partial x}\right) \\
=\left(\frac{2}{b-a}\right)^{1+v} C_{1+v} \sum_{m=2}^{\mathcal{M}} \sum_{n=1}^{\mathcal{N}} u_{N}\left(x_{m}, t_{n}\right) \mathcal{T}_{n}^{\tau}(t)\left\{{ }_{-1}^{R L} \mathcal{D}_{\xi}^{v}+{ }^{R L} \mathcal{D}_{1}^{v}\right\} \sum_{j=0}^{\mathcal{M}} \widetilde{\beta}_{m j} \frac{\partial}{\partial \xi}\left\{P_{j}(\xi)\right\}, \\
=\left(\frac{2}{b-a}\right)^{1+v} C_{1+v} \sum_{m=2}^{\mathcal{M}} \sum_{n=1}^{\mathcal{N}} u_{N}\left(x_{m}, t_{n}\right) \mathcal{T}_{n}^{\tau}(t) \sum_{j=0}^{\mathcal{M}} \widetilde{\beta}_{m j} \mathcal{W}_{j}^{v},
\end{gathered}
$$


in which we evaluate $\mathcal{W}_{j}^{v} \equiv\left(\frac{j+1}{2}\right)\left\{{ }_{-1}^{R L} \mathcal{D}_{\xi}^{v}+{ }_{\xi}^{R L} \mathcal{D}_{1}^{v}\right\} P_{j-1}^{1,1}(\xi)$ exactly by representing the Jacobi polynomial $P_{j-1}^{1,1}(\xi)$ as the following two alternative forms

$$
\begin{aligned}
P_{j-1}^{1,1}(\xi) & =\sum_{q=0}^{j-1} C_{j}(-1)^{q+j-1}\left(\frac{1}{2}\right)^{q}(1+\xi)^{q} \\
& =\sum_{q=0}^{j-1} C_{j}\left(\frac{-1}{2}\right)^{q}(1-\xi)^{q}
\end{aligned}
$$

where $\mathcal{C}_{j}=\left(\begin{array}{c}j+1+q \\ q\end{array}\right)\left(\begin{array}{c}j \\ j-1-q\end{array}\right)$. Hence,

$$
\begin{aligned}
\mathcal{W}_{j}^{v} & =\left(\frac{j+1}{2}\right)\left({ }_{a}^{R L} \mathcal{D}_{x}^{v(x, t)}+{ }_{x}^{R L} \mathcal{D}_{b}^{v(x, t)}\right)\left\{P_{j-1}^{1,1}(\xi)\right\} \\
& =\left(\frac{j+1}{2}\right)^{R L}{ }_{a} \mathcal{D}_{x}^{v(x, t)}\left\{\sum_{q=0}^{j-1} \mathcal{C}_{j}(-1)^{q+j-1}\left(\frac{1}{2}\right)^{q}(1+\xi)^{q}\right\} \\
& +\left(\frac{j+1}{2}\right)^{R L}{ }_{x} \mathcal{D}_{b}^{v(x, t)}\left\{\sum_{q=0}^{j-1} \mathcal{C}_{j}\left(\frac{-1}{2}\right)^{q}(1-\xi)^{q}\right\}
\end{aligned}
$$

by substituting $P_{j-1}^{1,1}(\xi)$ by (F.2) when taking the left-sided and by (F.3) when taking the right-sided Riemann-Liouville fractional derivative, respectively. Now, by (8) and (9), we obtain

$$
\begin{array}{r}
\mathcal{W}_{j}^{\nu}=\left(\frac{j+1}{2}\right) \sum_{q=\lceil\vee\rceil} \mathcal{C}_{j} \frac{\left(\frac{-1}{2}\right)^{q} \Gamma(q+1)}{\Gamma(q+1-v(x, t))}\left[(-1)^{j-1}(1+\xi)^{q-v(x, t)}\right. \\
\left.-(1-\xi)^{q-v(x, t)}\right] .
\end{array}
$$

Next, by substituting (F.4) into (F.1), evaluating it at the collocation points $\left(x_{i}, t_{k}\right)$, ans using the Kronecker delta property $\mathcal{T}_{n}^{\tau}\left(t_{k}\right)=\delta_{k n}$, we obtain

$$
\begin{aligned}
\frac{\partial^{1+v(x, t)} u_{N}(x, t)}{\left.\partial|x|^{1+v(x, t)}\right|_{\left(x_{i}, t_{k}\right)}} & =\left[\left(\frac{2}{b-a}\right)^{1+v} C_{1+v}\right]_{\left(x_{i}, t_{k}\right)} \sum_{m=2}^{\mathcal{M}} u_{N}\left(x_{m}, t_{k}\right) \sum_{j=1}^{\mathcal{M}} \widetilde{\beta}_{m j} \mathcal{W}_{j}^{v} \\
& =\sum_{m=2}^{\mathcal{M}}\left\{\mathbf{D}_{\text {Riesz }}^{1+v(x, t)}\right\}_{i k m} u_{N}\left(x_{m}, t_{k}\right),
\end{aligned}
$$


in which $\left\{\mathbf{D}_{\text {Riesz }}^{1+v(x, t)}\right\}_{i k m}$ denotes the $x$ - and $t$-dependent fractional diffusion differentiation matrix of Riesz type, give by

$$
\left\{\mathbf{D}_{\text {Riesz }}^{1+v(x, t)}\right\}_{i k m}=\left[\left(\frac{2}{b-a}\right)^{1+v} C_{1+v}\right]_{\left(x_{i}, t_{k}\right)} \sum_{j=1}^{\mathcal{M}} \widetilde{\beta}_{m j} \mathcal{W}_{j}^{v}(x, t) .
$$

\section{Acknowledgements}

This work was supported by the Collaboratory on Mathematics for Mesoscopic Modeling of Materials (CM4) at PNNL funded by the Department of Energy, by an AFOSR MURI, and by NSF/DMS.

\section{References}

[1] M. Zayernouri, G. E. Karniadakis, Fractional Sturm-Liouville EigenProblems: Theory and Numerical Approximations, Journal of Computational Physics 47-3 (2013) 2108-2131.

[2] D. A. Benson, S. W. Wheatcraft, M. M. Meerschaert, Application of a fractional advection-dispersion equation, Water Resources Research 36 (6) (2000) 1403-1412.

[3] F. Mainardi, Fractional calculus and waves in linear viscoelasticity: an introduction to mathematical models, Imperial College Press, 2010.

[4] W. Chester, Resonant oscillations in closed tubes, J. Fluid Mech 18 (1) (1964) 44-64.

[5] J. J. Keller, Propagation of simple non-linear waves in gas filled tubes with friction, Zeitschrift für angewandte Mathematik und Physik ZAMP 32 (2) (1981) 170-181.

[6] N. Sugimoto, T. Kakutani, Generalized Burgers' equation for nonlinear viscoelastic waves, Wave motion 7 (5) (1985) 447-458.

[7] R. L. Magin, Fractional Calculus in Bioengineering, Redding, CT: Begell House Inc., 2006.

[8] J. P. Bouchaud, A. Georges, Anomalous diffusion in disordered media: statistical mechanisms, models and physical applications, Physics reports 195 (4) (1990) 127-293. 
[9] R. Metzler, J. Klafter, The random walk's guide to anomalous diffusion: a fractional dynamics approach, Physics reports 339 (1) (2000) 1-77.

[10] R. Klages, G. Radons, I. M. Sokolov, Anomalous Transport: Foundations and Applications, Wiley-VCH, 2008.

[11] N. Sugimoto, Burgers equation with a fractional derivative; hereditary effects on nonlinear acoustic waves, J. Fluid Mech 225 (631-653) (1991) 4.

[12] E. Barkai, R. Metzler, J. Klafter, From continuous time random walks to the fractional Fokker-Planck equation, Physical Review E 61 (1) (2000) 132.

[13] B. Henry, S. Wearne, Fractional reaction-diffusion, Physica A: Statistical Mechanics and its Applications 276 (3) (2000) 448-455.

[14] T. Komatsu, On stable-like processes, Probability theory and mathematical statistics (Tokyo, 1995) (1995) 210-219.

[15] K. Kikuchi, A. Negoro, On Markov process generated by pseudodifferential operator of variable order .

[16] C. Coimbra, Mechanics with variable-order differential operators, Annalen der Physik 12 (11-12) (2003) 692-703.

[17] G. Cooper, D. Cowan, Filtering using variable order vertical derivatives, Computers \& geosciences 30 (5) (2004) 455-459.

[18] C. Tseng, Design of variable and adaptive fractional order FIR differentiators, Signal Processing 86 (10) (2006) 2554-2566.

[19] L. E. Ramirez, C. F. Coimbra, A variable order constitutive relation for viscoelasticity, Annalen der Physik 16 (7-8) (2007) 543-552.

[20] H. Pedro, M. Kobayashi, J. Pereira, C. Coimbra, Variable order modeling of diffusive-convective effects on the oscillatory flow past a sphere, Journal of Vibration and Control 14 (9-10) (2008) 1659-1672.

[21] H. G. Sun, W. Chen, Y. Q. Chen, Variable-order fractional differential operators in anomalous diffusion modeling, Physica A: Statistical Mechanics and its Applications 388 (21) (2009) 4586-4592. 
[22] C.-M. Chen, F. Liu, V. Anh, I. Turner, Numerical schemes with high spatial accuracy for a variable-order anomalous subdiffusion equation, SIAM Journal on Scientific Computing 32 (4) (2010) 1740-1760.

[23] C. M. Chen, F. Liu, V. Anh, I. Turner, Numerical simulation for the variableorder Galilei invariant advection diffusion equation with a nonlinear source term, Applied Mathematics and Computation 217 (12) (2011) 5729-5742.

[24] H. Zhang, F. Liu, M. S. Phanikumar, M. M. Meerschaert, A novel numerical method for the time variable fractional order mobile-immobile advectiondispersion model, Computers \& Mathematics with Applications .

[25] W. Chen, J. Zhang, J. Zhang, A variable-order time-fractional derivative model for chloride ions sub-diffusion in concrete structures, Fractional Calculus and Applied Analysis 16 (1) (2013) 76-92.

[26] P. Zhuang, F. Liu, V. Anh, I. Turner, Numerical methods for the variableorder fractional advection-diffusion equation with a nonlinear source term, SIAM Journal on Numerical Analysis 47 (3) (2009) 1760-1781.

[27] S. Shen, F. Liu, J. Chen, I. Turner, V. Anh, Numerical techniques for the variable order time fractional diffusion equation, Applied Mathematics and Computation 218 (22) (2012) 10861-10870.

[28] C. Chen, F. Liu, K. Burrage, Y. Chen, Numerical methods of the variableorder Rayleigh-Stokes problem for a heated generalized second grade fluid with fractional derivative, IMA Journal of Applied Mathematics (2012) 121.

[29] S. Shen, F. Liu, V. Anh, I. Turner, J. Chen, A characteristic difference method for the variable-order fractional advection-diffusion equation, Journal of Applied Mathematics and Computing (2013) 1-16.

[30] X. Zhao, Z. Sun, G. E. Karniadakis, Second-order approximations for variable order fractional derivatives: Algorithms and applications, Journal of Computational Physics, Accepted .

[31] Y. Lin, C. Xu, Finite difference/spectral approximations for the timefractional diffusion equation, Journal of Computational Physics 225 (2) (2007) 1533-1552. 
[32] X. Li, C. Xu, A space-time spectral method for the time fractional diffusion equation, SIAM Journal on Numerical Analysis 47 (3) (2009) 2108-2131.

[33] X. Li, C. Xu, Existence and uniqueness of the weak solution of the spacetime fractional diffusion equation and a spectral method approximation, Communications in Computational Physics 8 (5) (2010) 1016.

[34] M. M. Khader, On the numerical solutions for the fractional diffusion equation, Communications in Nonlinear Science and Numerical Simulation 16 (6) (2011) 2535-2542.

[35] C. Piret, E. Hanert, A radial basis functions method for fractional diffusion equations, J. Comp. Physics (2012) 71-81.

[36] E. Doha, A. Bhrawy, S. Ezz-Eldien, A Chebyshev spectral method based on operational matrix for initial and boundary value problems of fractional order, Computers \& Mathematics with Applications 62 (5) (2011) 23642373.

[37] A. H. Bhrawy, M. M. Al-Shomrani, A shifted Legendre spectral method for fractional-order multi-point boundary value problems, Advances in Difference Equations 2012 (1) (2012) 1-19.

[38] M. Maleki, I. Hashim, M. T. Kajani, S. Abbasbandy, An adaptive pseudospectral method for fractional order boundary value problems, in: Abstract and Applied Analysis, vol. 2012, Hindawi Publishing Corporation, 2012.

[39] D. Baleanu, A. Bhrawy, T. Taha, Two efficient generalized Laguerre spectral algorithms for fractional initial value problems, in: Abstract and Applied Analysis, vol. 2013, Hindawi Publishing Corporation, 2013.

[40] A. Bhrawy, M. Alghamdia, A New Legendre spectral Galerkin and pseudospectral approximations for fractional initial value problems 2013.

[41] W. Deng, J. Hesthaven, Local discontinuous Galerkin methods for fractional diffusion equations, ESAIM: Mathematical Modelling and Numerical Analysis (2013) 1845-1864.

[42] Q. Xu, J. Hesthaven, Stable multi-domain spectral penalty methods for fractional partial differential equations, Journal of Computational Physics 257 (2014) 241-258. 
[43] M. Zayernouri, G. E. Karniadakis, Exponentially accurate spectral and spectral element methods for fractional ODEs, Journal of Computational Physics 257-Part A (2014) 460 - 480.

[44] M. Zayernouri, G. E. Karniadakis, Discontinuous spectral element methods for time-and space-fractional advection equations, SIAM Journal on Scientific Computing 36 (4) (2014) B684-B707.

[45] M. Zayernouri, W. Cao, Z. Zhang, G. E. Karniadakis, Spectral and Discontinuous Spectral Element Methods for Fractional Delay Equations, SIAM Journal on Scientific Computing 36 (6) (2014) B904-B929.

[46] M. Zayernouri, G. E. Karniadakis, Fractional Spectral Collocation Method, SIAM Journal on Scientific Computing 36 (1) (2014) A40-A62.

[47] M. Zayernouri, M. Ainsworth, G. E. Karniadakis, A unified Petrov-Galerkin spectral method for fractional PDEs, Computer Methods in Applied Mechanics and Engineering, (2014) http://dx.doi.org/10.1016/j.cma.2014.10.051.

[48] I. Podlubny, Fractional Differential Equations, San Diego, CA, USA: Academic Press, 1999.

[49] R. Askey, J. Fitch, Integral Representations for Jacobi Polynomials and Some Applications, Journal of Mathematical Analysis and Applications 26 (1969) 411-437.

[50] G. Szegö, Orthongonal Polynomials, vol. 23, AMS Bookstore, 1992. 